\title{
Analysis and Prediction of COVID-19 using SIR, SEIR, and Machine Learning Models: Australia, Italy, and UK Cases
}

\author{
Iman Rahimi \\ Universiti Putra Malaysia \\ Amir H Gandomi ( $\sim$ gandomi@uts.edu.au ) \\ Data Science Institute, University of Technology Sydney, Australia \\ Fang Chen \\ Data Science Institute, University of Technology Sydney, Australia
}

\section{Research Article}

Keywords: COVID-19, Analysis, Machine learning, SIR and SEIR models, Optimization

Posted Date: October 13th, 2020

DOI: https://doi.org/10.21203/rs.3.rs-85513/v1

License: (c) (1) This work is licensed under a Creative Commons Attribution 4.0 International License.

Read Full License 


\title{
Analysis and Prediction of COVID-19 using SIR, SEIR, and Machine Learning Models: Australia, Italy, and UK Cases
}

\author{
Iman Rahimi ${ }^{1}$, Amir H Gandomi ${ }^{2}$, , Fang Chen ${ }^{2}$
}

4 (1)

Abstract- The novel Coronavirus disease, known as COVID-19, is an outbreak that started in Wuhan, one of the Central Chinese cities. In this report, a short analysis focusing on Australia, Italy, and the United Kingdom has been conducted. The analysis includes confirmed and recovered cases and deaths, the growth rate in Australia as compared with Italy and the United Kingdom, and the outbreak in different Australian cities. Mathematical approaches based on the susceptible, infected, and recovered case (SIR) and susceptible, exposed, infected, and recovered (SEIR) models were proposed to predict the epidemiology in the countries. Since the performance of the classic form of SIR and SEIR depends on parameter settings, some optimization algorithms, namely, the Broyden-Fletcher-Goldfarb-Shanno (BFGS), conjugate gradients (CG), L-BFGS-B, and Nelder-Mead are proposed to optimize the parameters of SIR and SEIR models and improve its predictive capabilities. The results of optimized SIR and SEIR models are compared with the Prophet algorithm and logistic function as two known ML algorithms. The results show that different algorithms display different behaviours in different countries. However, the improved version of the SIR and SEIR models have a better performance compared with other mentioned algorithms described in this study. Moreover, the Prophet algorithm works better for Italy and the United Kingdom cases than for Australian cases and Logistic function compared with Prophet algorithm has a better performance in these cases. It seems that Prophet algorithm is suitable for data with increasing trend in pandemic situations. Optimization of the SIR and SEIR models parameters has yielded a significant improvement in the prediction accuracy of the models. Although there are several algorithms for prediction of this Pandemic, there is no certain algorithm that would be the best one for all cases.

\footnotetext{
${ }^{1}$ Universiti Putra Malaysia, Malaysia

${ }^{2}$ Data Science Institute, University of Technology Sydney, Australia,

*corresponding author, email: gandomi@uts.edu.au
} 
Keywords- COVID-19, Analysis, Machine learning, SIR and SEIR models, Optimization.

27

Introduction: In December 2019, the Chinese government informed the rest of the world that a virus was spreading throughout China. A few months later, it spread very rapidly to some other countries. This virus is the Severe Acute Respiratory Syndrome- Related Coronavirus 2 which causes the disease novel coronavirus known as COVID-19. The United States Centers for Disease Control and Prevention (CDC) identified a seafood market in Wuhan that was suspected to be at the centre of the outbreak. The World Health Organization (WHO) reported a case in Thailand on Jan 13, which was the first time it was identified outside China. On Jan 16, Japan confirmed its first case of this novel coronavirus. On Jan 20, South Korea identified its first confirmed case of the new coronavirus. Nowadays, most countries in the world are affected by this virus.

Putra and Khozin Mu'tamar (2019) used Particle Swarm Optimization (PSO) algorithm to estimate parameters (Susceptible, Infected, Recovered) in the SIR model. The results indicate that the suggested method is precise enough with low error compared to analytical methods. Mbuvha and Marwala (2020) calibrated the SIR model to South Africa after considering different scenarios for R0 (reproduction number) for reporting infections and healthcare resource estimation for the next few days. Qi, Xiao et al. (2020) proposed that both daily temperature and relative humid-ity influenced the occurrence of COVID-19 in Hubei province and insome other provinces.

Salgotra, Gandomi et al. (2020) developed two COVID-19 prediction models based on genetic programming and applied this model in India. Findings from a study by (Salgotra, Gandomi et al. 2020) show genetic evolutionary programming models are highly reliable for COVID-19 cases in India.

In January 2020, the first case of Covid-19 was reported in Australia. In this report, a short analysis focusing on Australia was addressed and reported and continued as a simulation for the next few days.

The manuscript is organized in several sections. Section I presents the research methodology. Section II and III introduce the SIR and SEIR models. Section IV shows the prediction algorithms (logistic 
51 function and Prophet algorithm). Sections V shows the results. The conclusion and discussion are 52 provided in the last section.

62

63

64

65

66

67

68

69

70

71

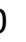
61

\section{Research methodology}

The study was carried out in several phases. For the first step, data were collected from World Health Organization (WHO) and John Hopkins University since they collect data from different organizations. After that, data were analyzed and preprocessed in order to avoid any duplicated and missing values. Numerical tests were performed using Python and $\mathrm{R}$ and executed on a computer Intel ${ }^{\circledR}$ Core i7-4510U $2.0 \mathrm{GHz} 8$ GB DDR3 Memory (Supplementary file). The flowchart of the research methodology is provided in Figure 1.

\section{Data Collection}

- Source: WHO
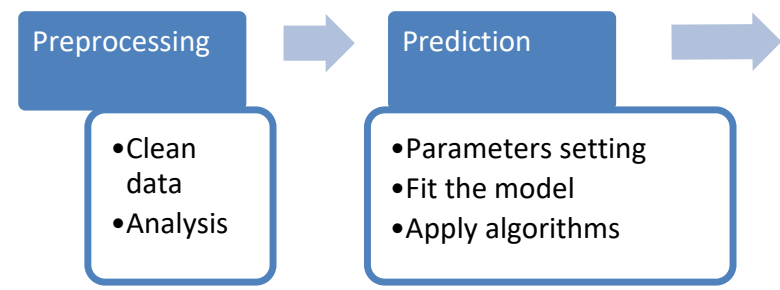

\section{Comparison}

- Performance metric

- Fit the mode

-Apply algorithms

What happened? What is going to happen in future?

Figure 1 Flowchart of the current research process

\section{The SIR model}

This section introduces the classic form of the SIR model (Kermack and McKendrick 1932, Capasso and Serio 1978) that is used to describe the transmission of COVID-19 virus in Australia, Italy, and the United Kingdom. The flowchart of SIR model is shown in Figure 2:

\section{Susceptible (S)}

\section{Infectious (I)}




$$
\begin{aligned}
& \frac{d s}{d t}=-\beta I S \\
& \frac{d I}{d t}=\beta I S-\gamma I \\
& \frac{d R}{d t}=\gamma I
\end{aligned}
$$

in which

- $\mathrm{S}$ is the number of individual susceptible at time $\mathrm{t}$.

- I is the number of infected individuals at time $t$.

- $\mathrm{R}$ is the number of recovered individuals at time $\mathrm{t}$.

- $\quad \beta$ and $\gamma$ are the transmission rate and rate of recovery (removal), respectively.

\section{The SEIR model}

The SEIR model is an extended version of SIR model (Peng, Yang et al. 2020). It models the interaction of people between different conditions: the susceptible (S), exposed (E), infective $(\mathrm{I})$, and recovered $(\mathrm{R})$. The parameters $\mathrm{S}, \mathrm{I}$, and R are same as parameters in SIR model and $\mathrm{E}$ presents the fraction of individuals that have been infected but does not show any signs. The SEIR-model diagram is as follows (Fig. 3): 


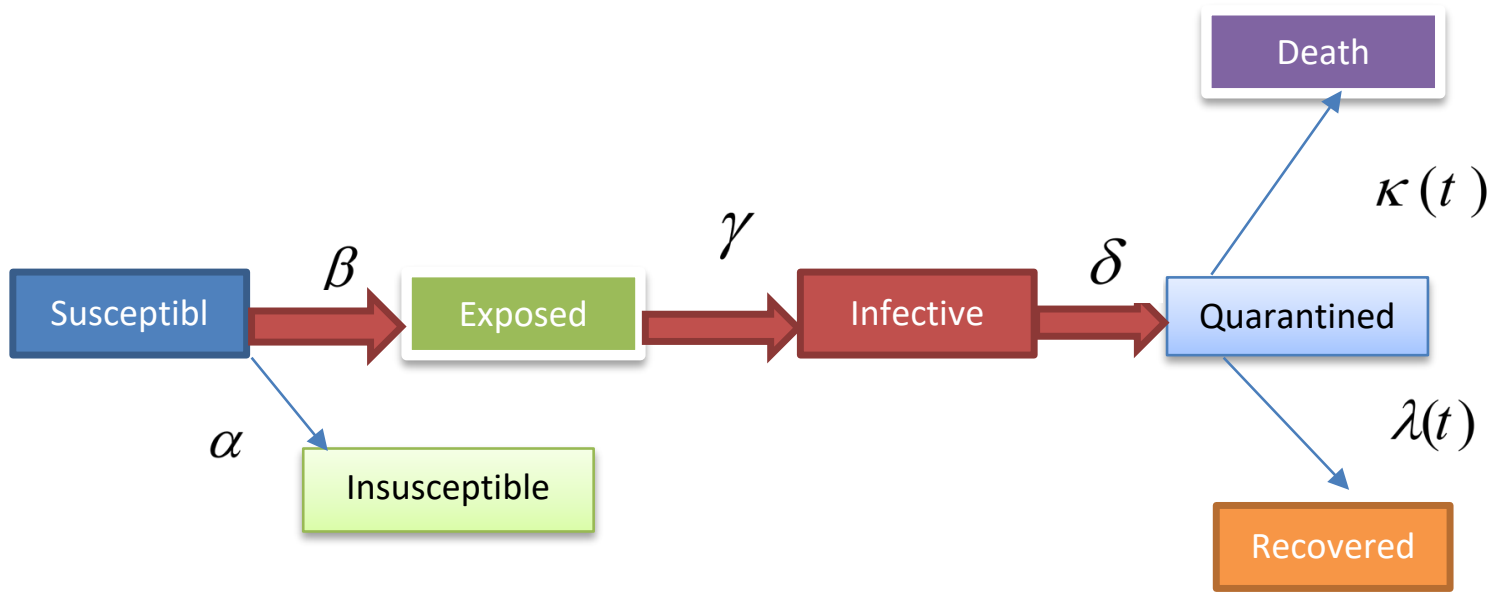

91

$$
\begin{aligned}
& \frac{d S(t)}{d t}=-\beta \frac{S(t) I(t)}{N}-\alpha S(t) \\
& \frac{d E(t)}{d t}=\beta \frac{S(t) I(t)}{N}-\gamma E(t) \\
& \frac{d I(t)}{d t}=\gamma E(t)-\delta I(t) \\
& \frac{d Q(t)}{d t}=\delta I(t)-\lambda(t) Q(t)-\kappa(t) Q(t) \\
& \frac{d R(t)}{d t}=\lambda(t) Q(t) \\
& \frac{d D(t)}{d t}=\kappa(t) Q(t) \\
& \frac{d P(t)}{d t}=\alpha S(t)
\end{aligned}
$$

92

Where

$\alpha$ presents the protection rate, $\beta$ shows the infection rate, illustrates the inverse of the

average latent time, $\delta$ displays the inverse of the average quarantine time, $\lambda_{0}$ and $\lambda_{1}$ are 
coefficients used in the time- dependent cure rate, $\kappa_{0}$ and $\kappa_{1}$ are coefficients used in the timedependent mortality rate (Peng, Yang et al. 2020).

\section{Prediction}

In the present section, some machine learning techniques were used for COVID-19 case predictions in Australia, Italy, and the United Kingdom. Machine learning is a branch of computer science in which data could teach algorithms. The learning process could be done as supervised-, unsupervised, and/or semi-supervised learning forms (Mitchell 1997, Arkes 2001, Armstrong 2001, Nikolopoulos, Litsa et al. 2015, Maleki, Mahmoudi et al. 2020). In this section, some approaches that are used for prediction of cases (confirmed and deaths) of COVID-19 Pandemic are provided.

\section{a) Logistic function}

A logistic function could be defined as follows:

$$
f(x)=\frac{L}{1+e^{-k\left(x-x_{0}\right)}}
$$

$\mathrm{e}=$ Euler's number

$$
x_{0}=\text { Sigmoid 's midpo int, }
$$

$\mathrm{L}$ is the curve's maximum value,

and $\mathrm{K}$ is the logistic growth of the curve

\section{b) Times Series forecasting with the Prophet algorithm}

The Prophet algorithm is an open-source tool developed by Facebook' s Data Science team, and its main goal is business forecasting (Taylor and Letham 2017, Taylor and Letham 2018). The Prophet algorithm works well with time-series data that have seasonal effects and are robust in dealing with missing data (Ndiaye, Tendeng et al. 
126 2020). In the Prophet algorithm, the forecast could be written as shown in Equation 5 (Ndiaye, Tendeng et al. 2020):

$$
\underset{\substack{\hat{y} \\ T+h T}}{\hat{y}}=\bar{y}=\left(y_{1}+y_{2}+\ldots y_{T}\right) / T
$$

in which $y_{1}, y_{2}, \ldots, y_{T}$ are denoted as historical data, and $\underset{T+h \mid T}{\hat{y}}$ is a short-hand to forecast $\underset{T+h \mid T}{y}$ based on available data.

\section{Results}

\section{a. Analysis}

\section{i. New cases}

In this sub-section, the confirmed growth rates focusing on Australia, Italy, and the United Kingdom for every day from 2020-04-24 to 2020-05-23 were calculated. Figure 4 depicts the growth rate of confirmed cases in the countries. As can be seen in Figure 4, the growth rate for Australia was always below 0.5 during times of outbreak and just above 0.0 at the of May, while the rate for Italy and the United Kingdom is generally high. The growth rate for the United Kingdom was almost above 2.0 in April and then dramatically declined in May. The rate for Italy fluctuates between 0.5 and 1.5 in April and May.

Figure 5 also presents the growth rate of death cases for the above-mentioned countries daily from 2020-04-24 to 2020-05-23. The growth rate for death cases in Australia fluctuated between 0 and 7 in April and May and was 7 at the end of April (higher than Italy and United Kingdom during the same time), while for Italy, the rate was almost below 2.0 during the same time period and for the United Kingdom, the rate was just below 4.0 at the end of April and just above 0.0 at the end of May. 


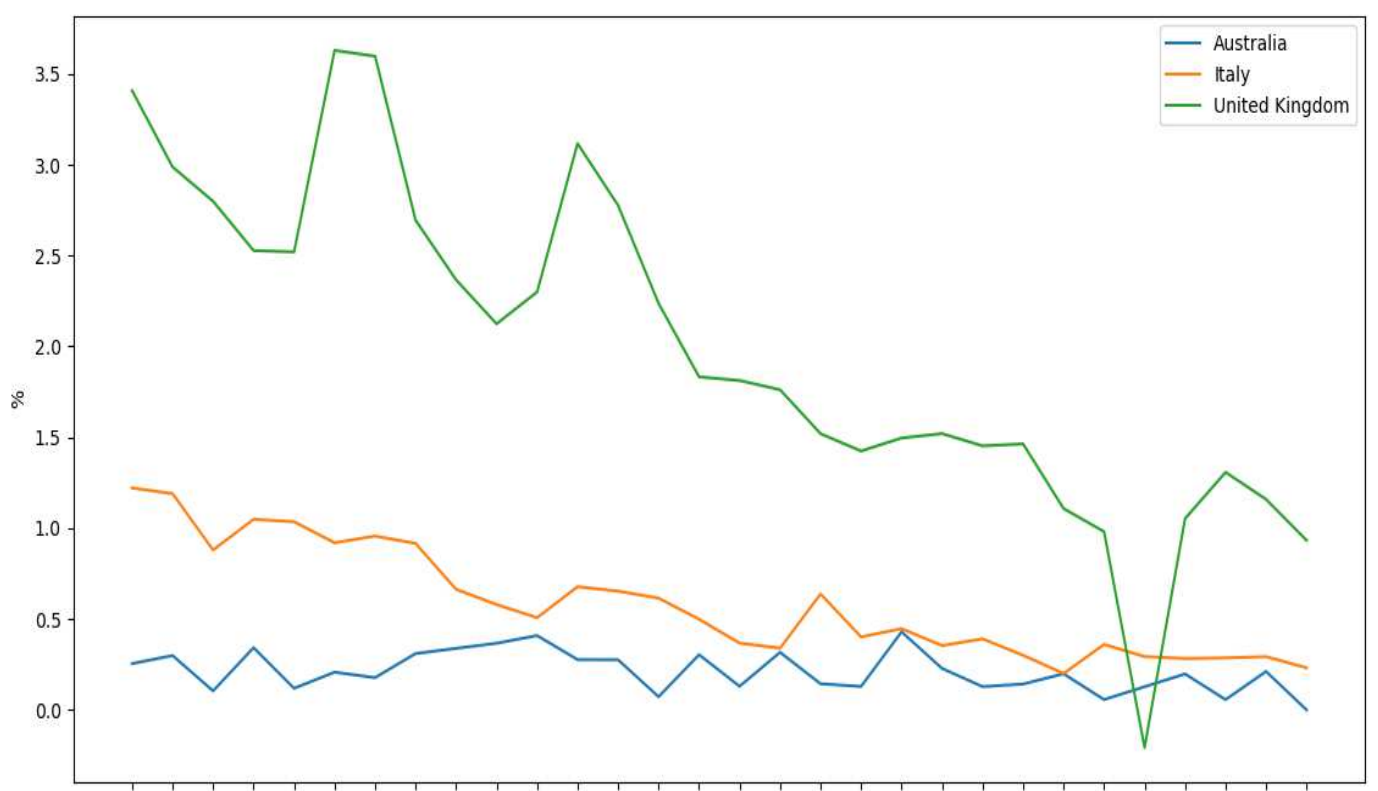

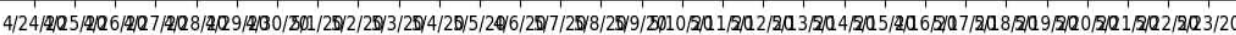
Growth_rate (Confirmed cases in Australia, Italy, and United Kingdom)

Figure 4 Growth rate (Confirmed cases in Australia, Italy, and the United Kingdom)

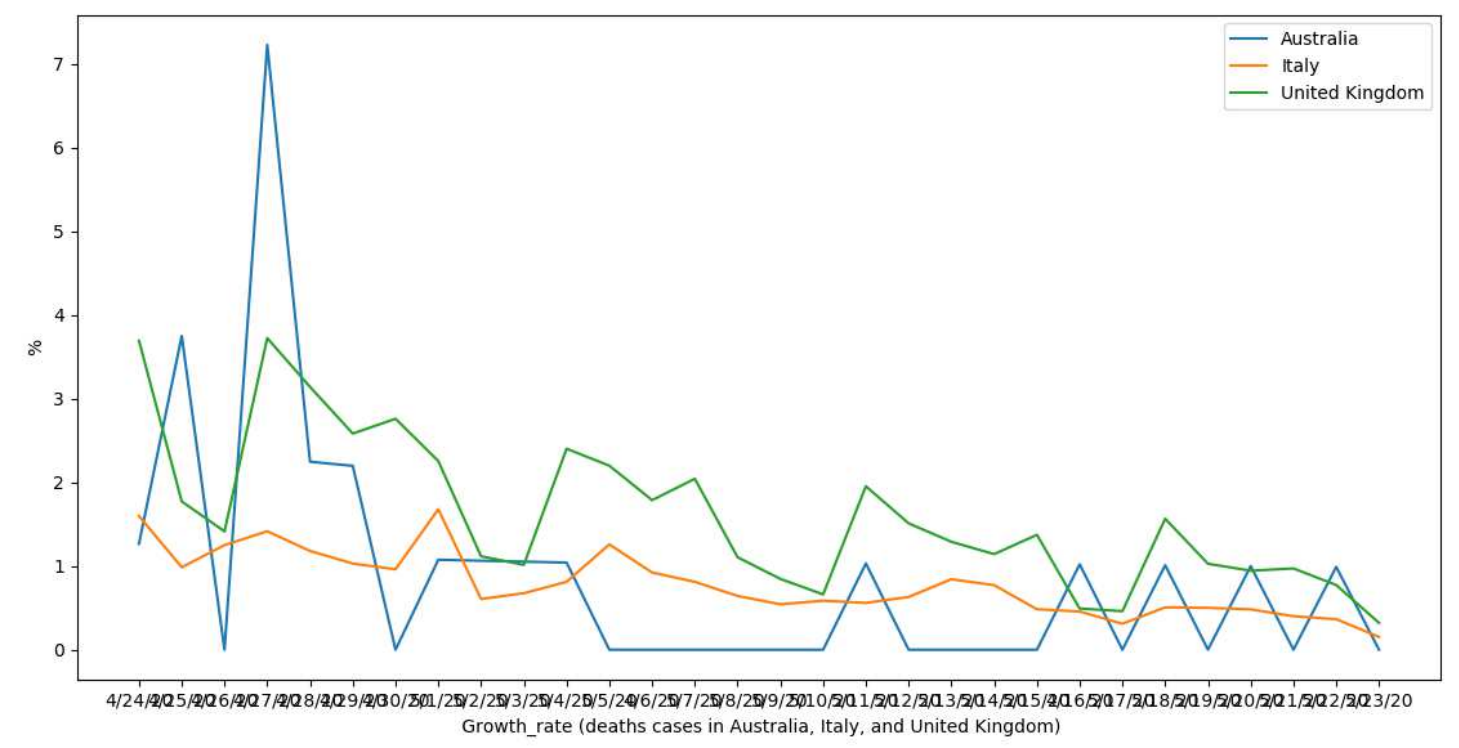

Figure 5 Growth rate (death cases in Australia, Italy, and the United Kingdom)

\section{ii. Overall growth rate}

148 This section shows numbers of active cases in these three countries. The active cases were calculated using the following equation: 
Active_cases=confirmed_cases - deaths_cases -recovered_cases

151

152 From equation (13), the overall growth rate could be calculated according to Equation 14:

Overall growth rate[i] $=(($ active cases[i]-active case[i-1] $) /$ active case $[\mathrm{i}-1]) * 100$

153 In equation (14), the index i presents day. Figure 6 illustrates the overall growth rate for confirmed 154 cases in the countries. Negative numbers show that people recovering are faster than those getting sick 155 and that would be good news. The rate for Australia in the time period was almost below zero and 156 changed from -15 at the end of April to just below -5 at the end of May and for Italy fluctuated between 157 just above -7.5 and just above 0.0 , while the rate for the United Kingdom was almost always positive 158 number in the time horizon (00.0 and 3.0). Figure 7 illustrates the number of death cases in Australia compared with the two other countries, and it is clear that the number in Australia is significantly lower 160 than other two. 


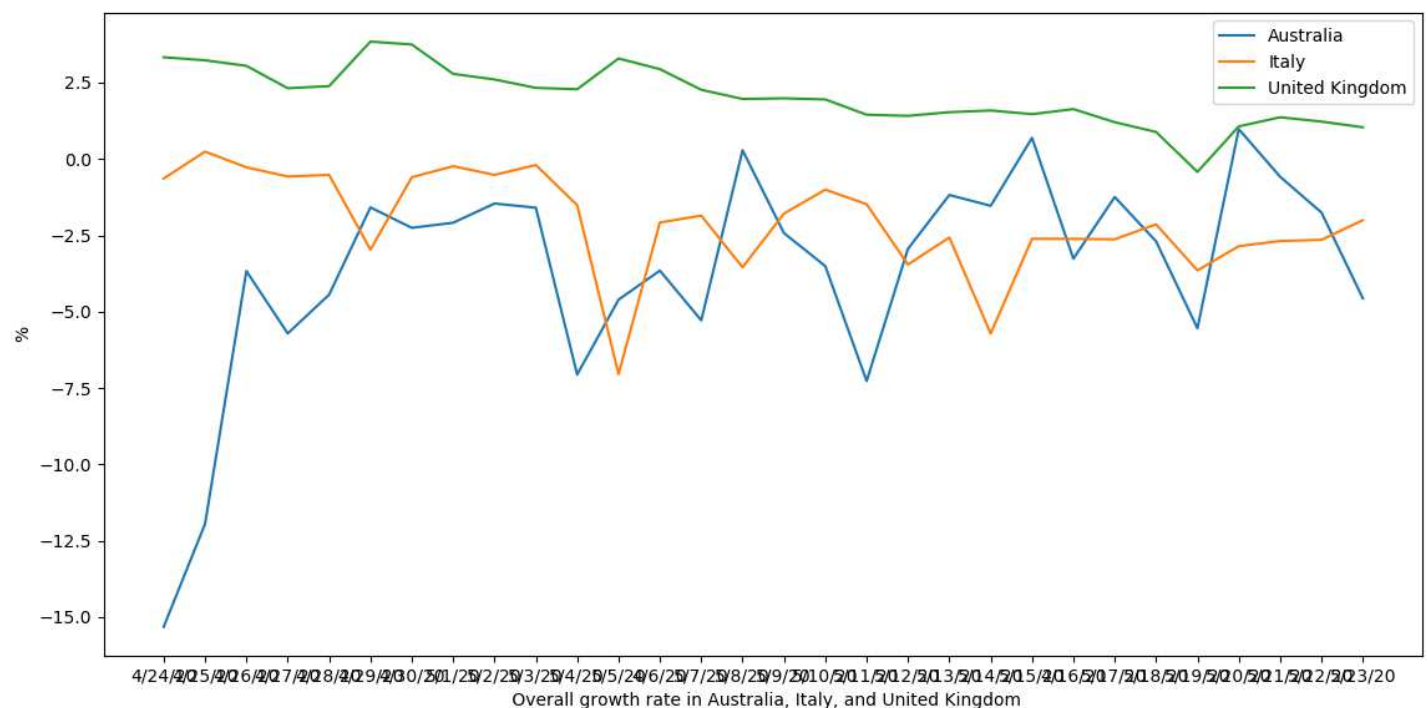

Figure 6 Overall growth rate for confirmed cases in Australia, Italy, and the United Kingdom

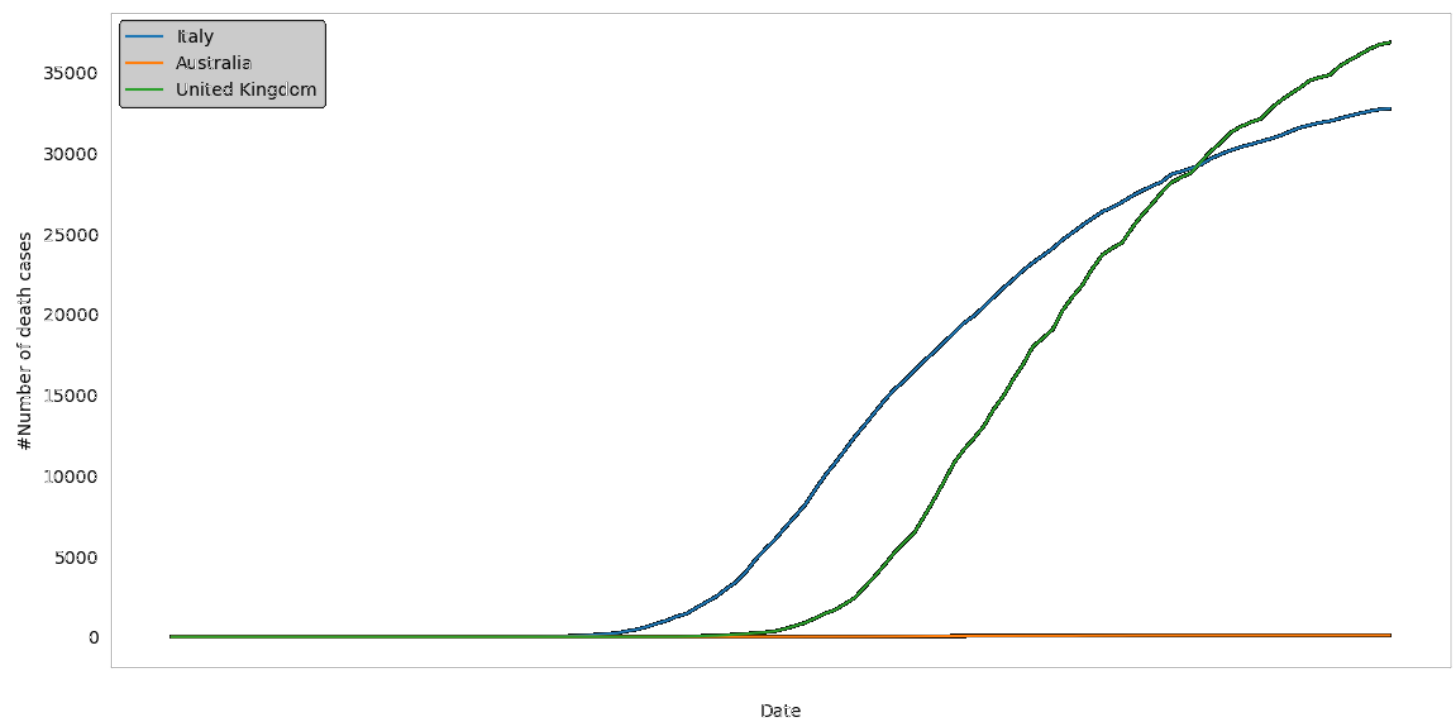

Figure 7 Number of death cases in Australia compared with Italy and the United Kingdom 


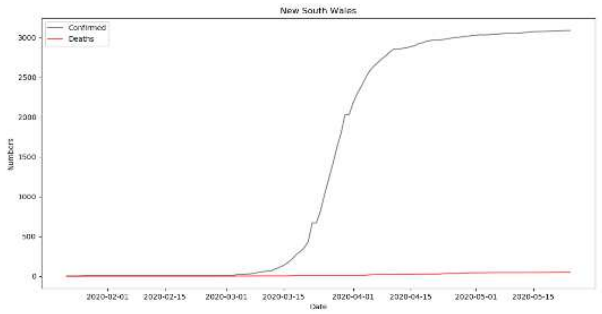

Figure 8 (a) New South Wales

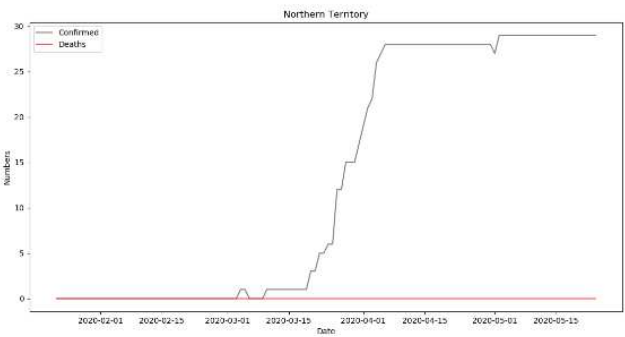

Figure 8(c) Northern Territory

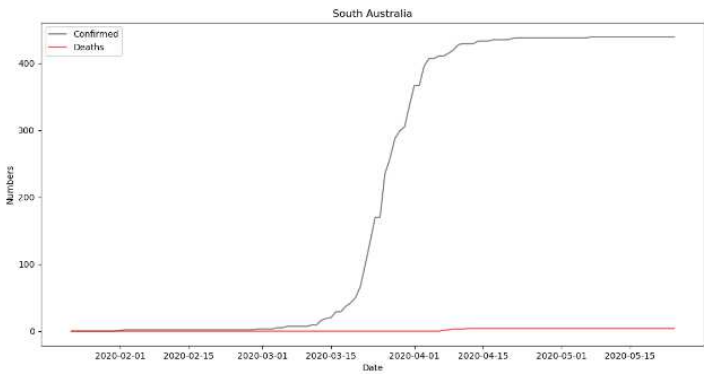

Figure 8(e) South Australia

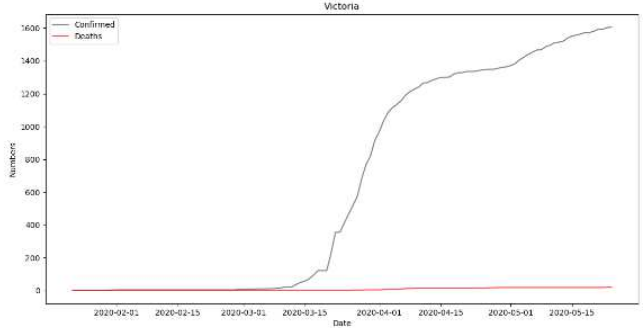

Figure $8(g)$ Victoria

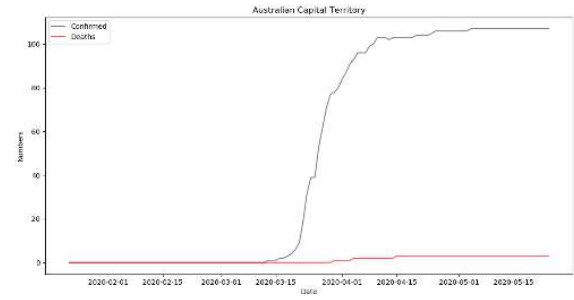

Figure 8 (b) Australian Capital Territory

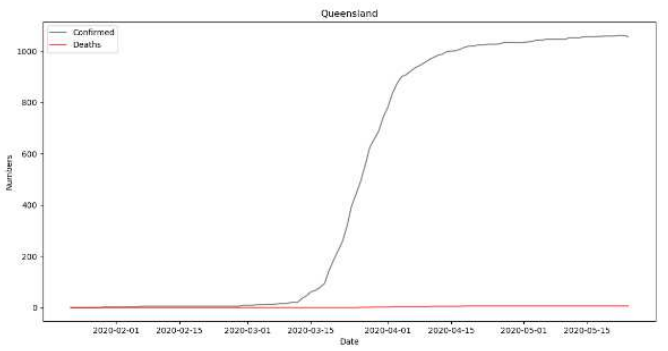

Figure 8(d) Queensland

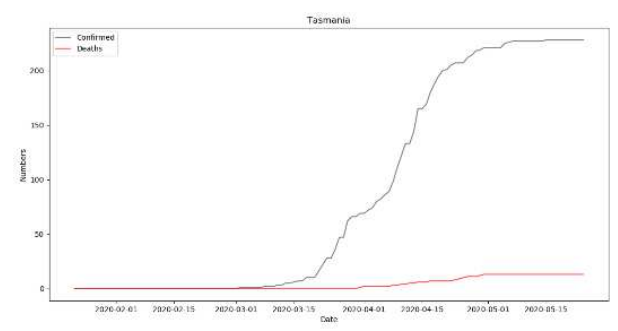

Figure 8(f) Tasmania

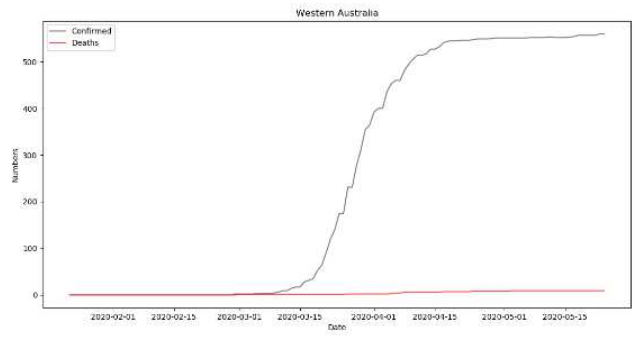

Figure 8(h) Western Australia

Figure $8(a-h)$ shows confirmed versus deaths cases in each individual Australian state. By now (202005-23), New South Wales and Northern Territory possess the most and least number of confirmed and death cases in Australia, respectively. From Figure $8(a-h)$, the number of confirmed and death cases in 
171 New South Wales significantly differed from other states in Australia and increased dramatically, while

172 the Northern Territory experienced some fluctuation during the study time period.

173

174 With the aim of forecasting, the logistic function is defined in Equation (11) and was applied to collected 175 data (Time horizon: start of outbreak in the countries) and results have been illustrated in Figures 9-14.

176 As it is shown in Figures 9-14, the logistic function is fitted until the trend of cases is increases and to 177 evaluate the performance of metric R2 scores used for confirmed and death cases. Results are presented 178 in Table 2. Another metric that has been used in experiments is the root mean square error (RMSE), 179 and the results of RMSE I depicted in Table 2. The best RMSE value belongs to the Australian cases 180 (confirmed and deaths).

Table 1 R2 score fore different countries, different cases

\begin{tabular}{|l|l|l|}
\hline countries & Confirmed cases & Deaths cases \\
\hline Australia & 0.87 & 0.67 \\
\hline United Kingdom & 0.92 & 0.97 \\
\hline Italy & 0.93 & 0.95 \\
\hline
\end{tabular}

182

Table 2 Root mean square error (RMSE) values for different countries and different cases

\begin{tabular}{|l|l|l|}
\hline countries & Confirmed cases & Deaths cases \\
\hline Australia & 8.22 & 0.88 \\
\hline United Kingdom & 21.94 & 6.97 \\
\hline Italy & 23.24 & 8.00 \\
\hline
\end{tabular}


Prediction of confirmed cases in Australia by logistic function

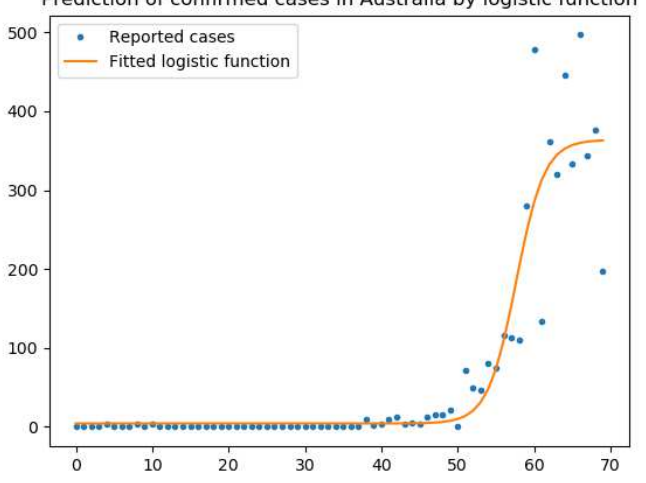

Figure 9 Prediction of confirmed cases by logstic function (Australia)

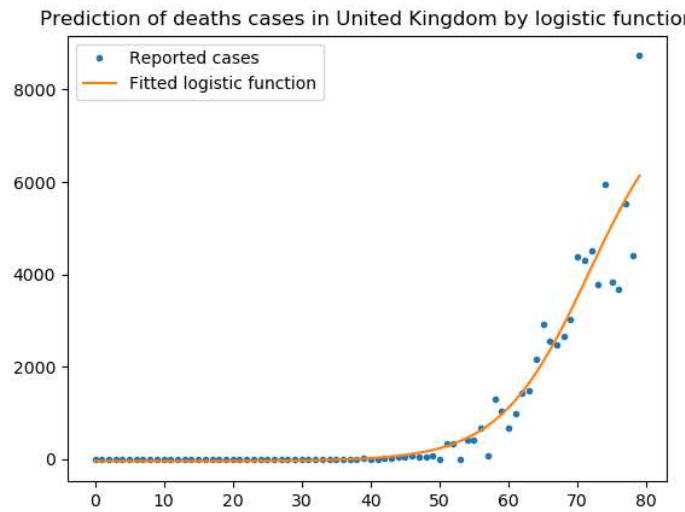

Figure 11 Prediction of confirmed cases by logstic function (United Kingdom)

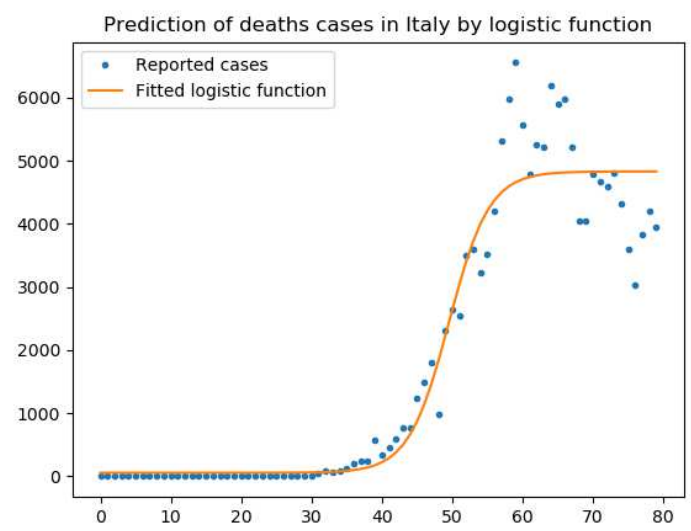

Figure 13 Prediction of confirmed cases by logstic function (Italy)

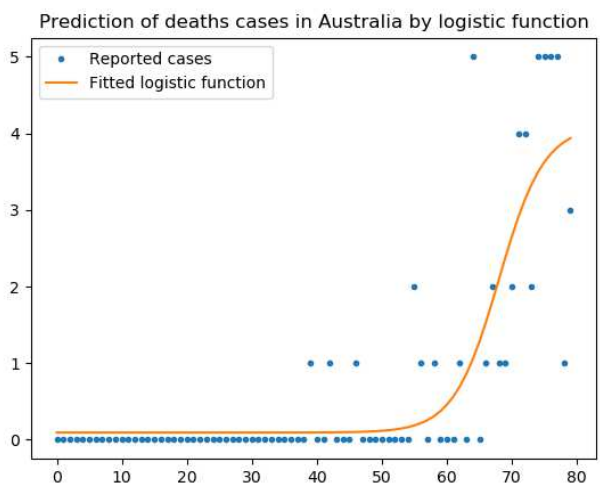

Figure 10 Prediction of death cases by logistic function (Australia)

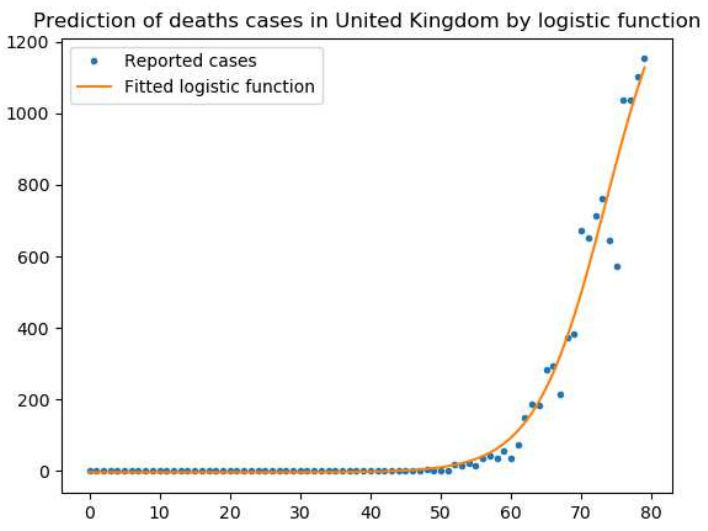

Figure 12 Prediction of deaths cases by logstic function (United Kingdom)

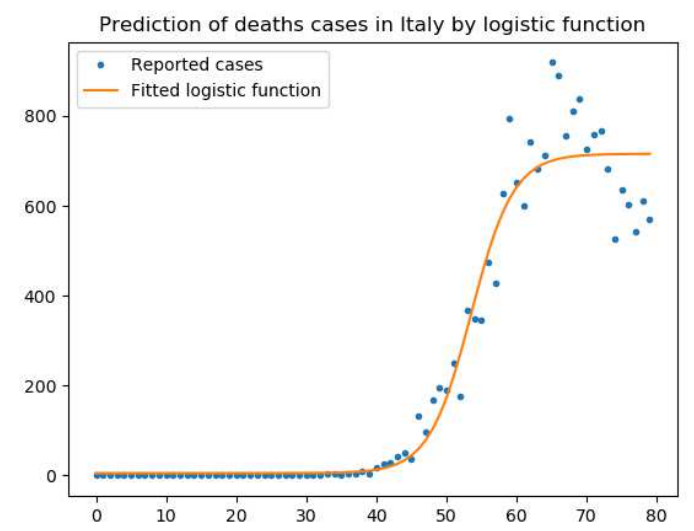

Figure 14 Prediction of deaths cases by logstic function (Italy) 
Figures 15-17 present the results of the classic SIR model. As previously mentioned, controlling $\beta$ parameters indicate the level of disease transmission, and $\gamma$ is the recovery (removal) period indicating how much peope could recover in a period. First, all parameters were initially added to the SIR model and applied it to real data, but it can be seen from Figures 15-17 and Table 3 (RMSE values) in which the classic form was not suitable for prediction of the COVID-19 pandemic in these three countries. In order to fit the SIR models to Australia, Italy, and the United Kingdom, an optimizer was needed to find the unknown parameters ( $\beta$ and $\gamma)$ from equation $R_{0}\left(R_{0}=\frac{\beta}{\gamma}\right)$ since these parameters could be estimated. Before the start of the outbreak, it is essential to address whether the number of susceptible cases is equal to the number of people in these countries because no antibodies exist, and no vaccines for the disease have been developed. At first, $R_{0}=2.7$ was fixed (reported by Australian Government: Department of Health) as the the median number, $\beta=0.378$, and $\gamma=0.14$. Figure 19 (a-c) present the confirmed cases provided by the optimized SEIR model with the abovementioned decriptions in the three countries (See Figure 18).

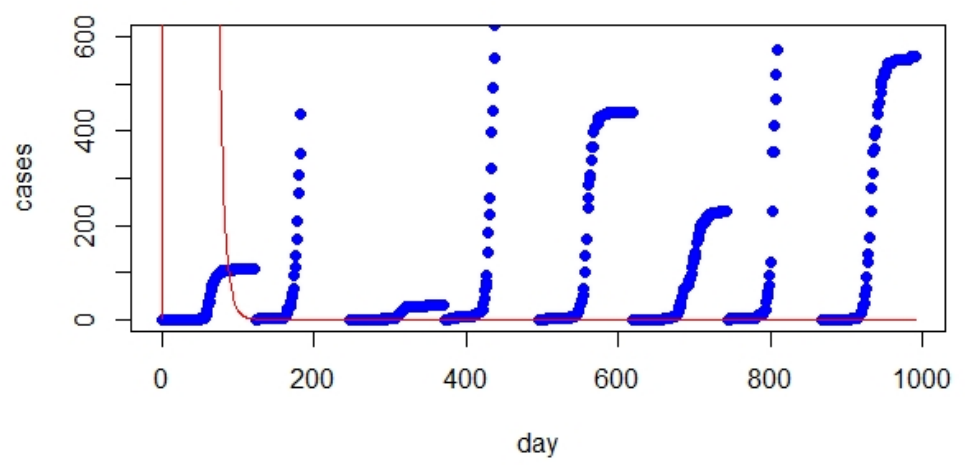

Figure 15 Predicted cases in Australia using the susceptible, infected, recovered (SIR) model (blue: real confirmed cases, red: SIR model) 


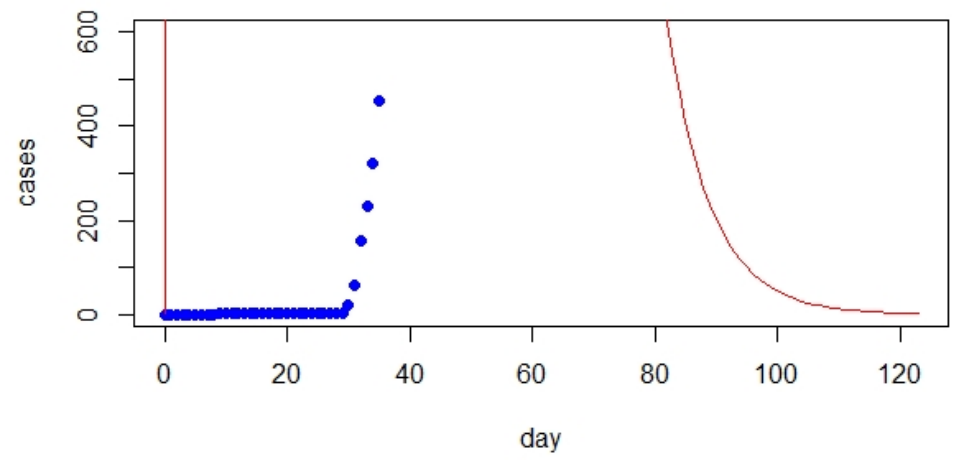

205

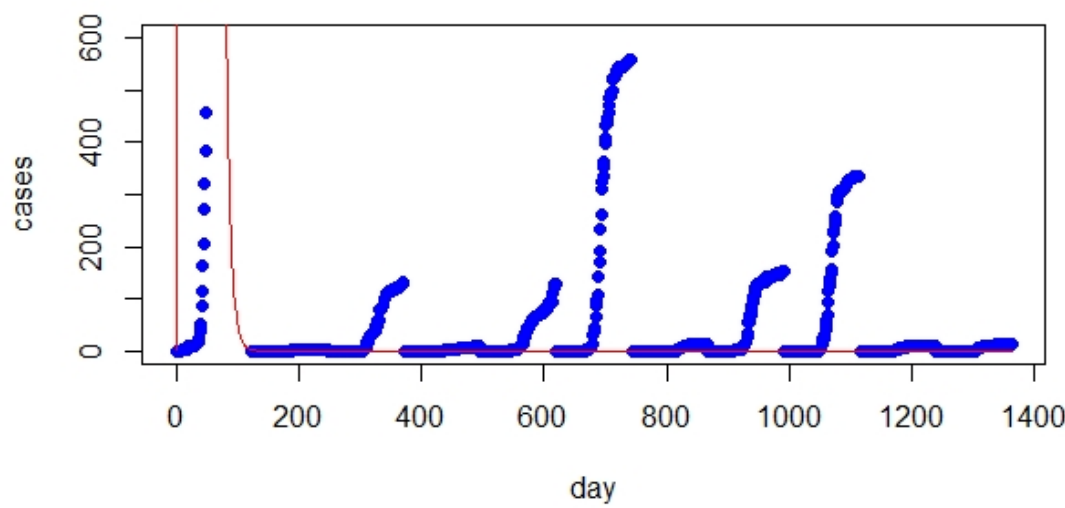

207

Figure 17 Predicted cases in UK based on the SIR model (blue: real confirmed cases, red: SIR model)

Table 3 RMSE values obtained by SIR model (before optimization of parameters)

\begin{tabular}{|l|l|l|}
\hline Italy & United Kingdom & Australia \\
\hline $\mathbf{1 8 . 7 5}$ & 15.45 & 831.84 \\
\hline
\end{tabular}

Real data were used to estimate the values of $\beta$ and $\gamma$. An optimizer was used to find the best estimation of $\beta$ and $\gamma$. The optimization algorithms were the Broyden-Fletcher-GoldfarbShanno (BFGS) algorithm (Fletcher 1987), L-BFGS-B (Byrd, Lu et al. 1995), conjugate gradients (CG), (Fletcher and Reeves 1964), and Nelder-Mead (Nelder and Mead 1965). The parameter settings are provided in Table 3. The flowchart of the improved SIR and SEIR 

and Table 4, respectively.

-Defining initial values for the parameters and variables.

-Solving the SIR and SEIR models, numerically.

\section{Step 1}

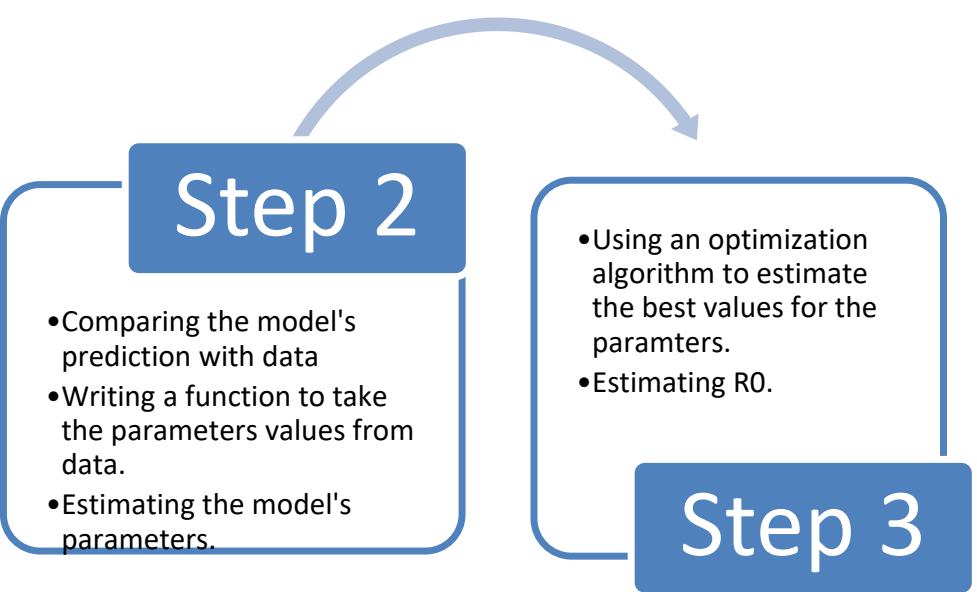

219

Figure 18 Flowchart of improved version of SIR and SEIR models

Table 4 Parameter settings

\begin{tabular}{|l|l|}
\hline Algorithm & Parameters setting \\
\hline BFGS & Maxit $=100$, reltol ${ }^{*}=1 \mathrm{e}^{-8}$ \\
\hline Nelder-Mead & Maxit $=500$, reltol $=1 \mathrm{e}^{-8}$, alpha $=1$, beta $=0.5$, gamma $=2.0$ \\
\hline $\begin{array}{l}\text { L-BFGS-B } \\
\text { CG }\end{array}$ & $\begin{array}{l}\text { Maxit }=100, \text { reltol }=1 \mathrm{e}^{-8}, 1 \mathrm{~mm}^{* *}=5, \text { factr } * * *=1 \mathrm{e}^{7} \\
\text { Maxit }=100, \text { reltol }=1 \mathrm{e}^{-8}\end{array}$ \\
\hline${ }^{*}$ Reltol= Relative convergence tolerance ${ }^{* *} \mid \mathrm{mm}=$ number of BFGS updates retained,$* * *$ factr=convergence factor \\
\hline
\end{tabular}

Table 5 shows the optimized values obtained by different algorithms (SIR model). The best values for the parameters were found using the Nelder-Mead algorithm (for SIR model) and L-BFGS-B algorithm (for SEIR model). This method is illustrated in Figure 18. As was mentioned earlier, before the start of the outbreak, the number of susceptible cases was equal to the number of people in these countries because no antibodies exist, and no vaccine for the disease is available. From Wikipedia, the populations of Australia, Italy, and the United Kingdom are $25^{06}, 60^{06}$, and $67^{06}$, respectively. Table 6 illustrates the RMSE values obtained by the algorithms (for SIR and SEIR models) showing improvements in significantly reducing the values. 
Table 5 Median values of SIR parameters determined by the departments of health in each country

$\begin{array}{llll}\text { COUNTRY } & \beta & \gamma & R_{0}\end{array}$

\begin{tabular}{|c|c|c|c|c|c|c|c|c|c|c|c|c|}
\hline Algorithm & BFGS & Nelder-Mead & L-BFGS-B & CG & BFGS & Nelder-Mead & L-BFGS-B & $\mathrm{CG}$ & BFGS & Nelder-Mead & L-BFGS-B & $\mathrm{CG}$ \\
\hline Australia & 0.014 & 0.014 & 0.378 & 0.37 & 0.22 & 0.22 & 0.14 & 0.14 & 0.063 & 0.063 & 2.64 & 2.64 \\
\hline United Kingdom & 0.37 & $3.84701^{-3}$ & 0.37 & 0.37 & 0.14 & $1.94^{-1}$ & 0.14 & 0.14 & 2.64 & 0.02 & 2.64 & 2.64 \\
\hline Italy & 0.37 & $1.083555^{-3}$ & 0.37 & 0.37 & 0.14 & $3.9088^{-1}$ & 0.14 & 0.37 & 2.64 & 0.01 & 2.64 & 2.64 \\
\hline
\end{tabular}

236

Table 6 RMSE values obtained based on the improved SIR model considering a 0.99 confidence interval

\begin{tabular}{|l|l|l|l|}
\hline Model & Italy & United Kingdom & Australia \\
\hline SIR model & 1.41 & 1.01 & 1.13 \\
\hline SEIR model & 1.12 & 1.23 & 1.04 \\
\hline
\end{tabular}

238 


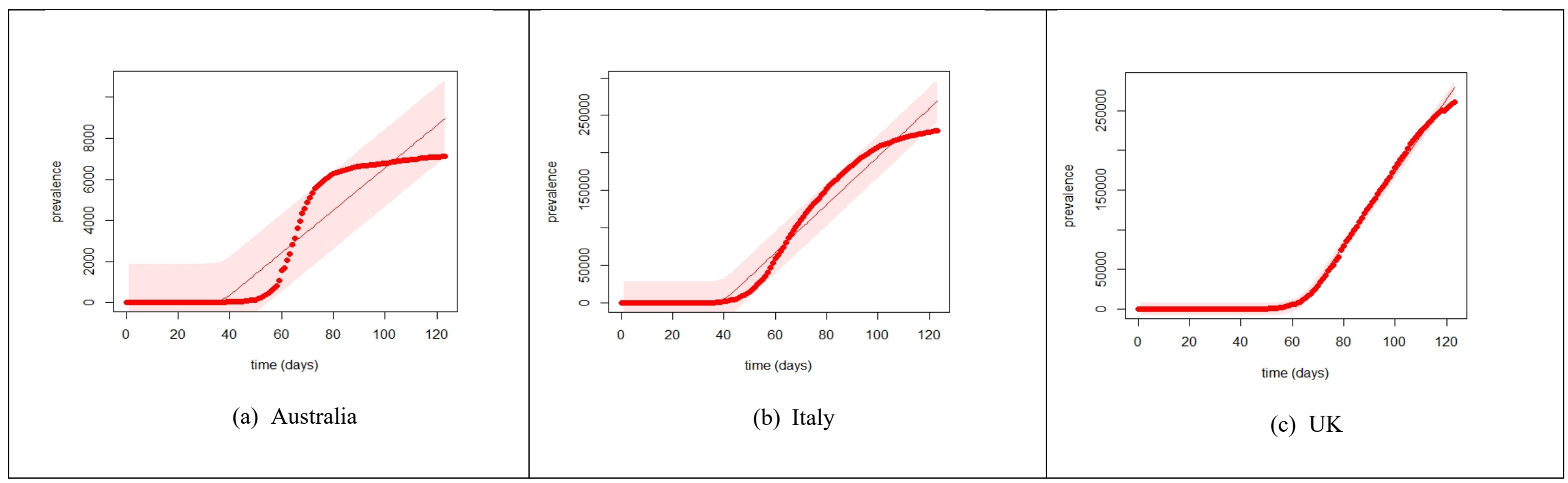


Table 7 Predicted cumulative confirmed cases in Australia (cross-validation matrix)

\begin{tabular}{|c|c|c|c|c|c|}
\hline $\mathbf{y}$ & $\mathbf{d s}$ & $\hat{y}$ & $\hat{y_{\text {lower }}}$ & $\hat{y}_{\text {upper }}$ & cutoff \\
\hline 7095 & $2020-05-21$ & 21309.752 & 18998.140 & 23829.955 & $2020-04-04$ \\
\hline 7099 & $2020-05-22$ & 21630.708 & 19245.072 & 24269.904 & $2020-04-04$ \\
\hline 7114 & $2020-05-23$ & 21959.985 & 19424.097 & 24640.939 & $2020-04-04$ \\
\hline 7114 & $2020-05-24$ & 22326.688 & 19766.194 & 25093.353 & $2020-04-04$ \\
\hline
\end{tabular}

245

Table 8 Predicted cumulative confirmed cases in the United Kingdom (cross-validation matrix)

\begin{tabular}{|c|c|c|c|c|c|}
\hline $\mathbf{y}$ & $\mathbf{d s}$ & $\hat{y}$ & $y_{\text {lower }}$ & $\hat{y}_{\text {upper }}$ & cutoff \\
\hline 252246 & $2020-05-21$ & 143776.53 & 126702.28 & 162413.93 & $2020-04-04$ \\
\hline 255544 & $2020-05-22$ & 146462.83 & 128526.68 & 165539.80 & $2020-04-04$ \\
\hline 258504 & $2020-05-23$ & 148818.88 & 130813.85 & 168216.41 & $2020-04-04$ \\
\hline 260916 & $2020-05-24$ & 150344.39 & 131476.87 & 170004.00 & $2020-04-04$ \\
\hline
\end{tabular}

247

248 Tables 7-9 present the results of the predicted cumulative confirmed cases obtained using the Prophet

249 algorithm in the three countries. In the presented tables, $\mathbf{y}$ represents the true values of confirmed cases,

250 ds is time, $y$ is the forecasted values, $y_{\text {lower }}$ and $y_{\text {upper }}$ are the lower and upper bounds for the

251 forecasted values, respectively. It should be noted, the forecasted values were made between the cutoff

252 and cutoff + horizon. Tables 7-9 are also called cross-validation matrices that are used to find the error

253 values between $\mathbf{y}$ and ${ }^{y}$ after which the RMSE values can be obtained (Figure 23 a-c). Figures 20-22

254 visualize forcasted values obtained using the Prophet algorithm, indicating the mentioned algorithm is

255 fitted for the cases of Italy and the United Kingdom but with errors for Australia. 
Table 9 Predicted cumulative confirmed cases in Italy (cross-validation matrix)

\begin{tabular}{|c|c|c|c|c|c|}
\hline $\mathbf{y}$ & $\mathbf{d s}$ & $\hat{y}$ & $\hat{y_{\text {lower }}}$ & $\hat{y}_{\text {upper }}$ & cutoff \\
\hline 228006 & $2020-05-21$ & 373982.5 & 336940.1 & 415612.7 & $2020-04-04$ \\
\hline 228658 & $2020-05-22$ & 379300.7 & 340862.6 & 422338.4 & $2020-04-04$ \\
\hline 229327 & $2020-05-23$ & 384792.4 & 344957.8 & 429120.3 & $2020-04-04$ \\
\hline 229858 & $2020-05-24$ & 390481.8 & 349482.8 & 436663.2 & $2020-04-04$ \\
\hline
\end{tabular}

259

260

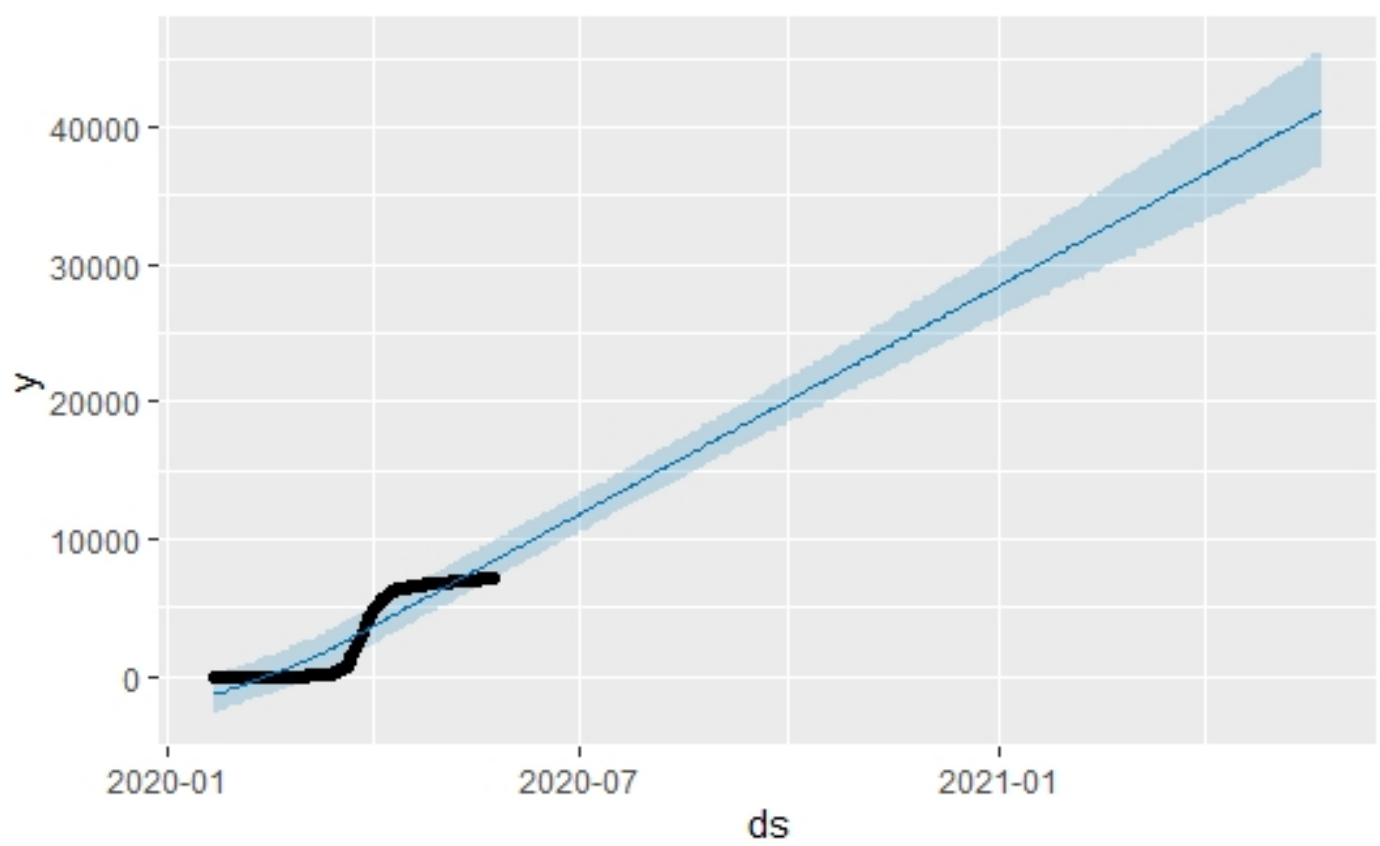

261 


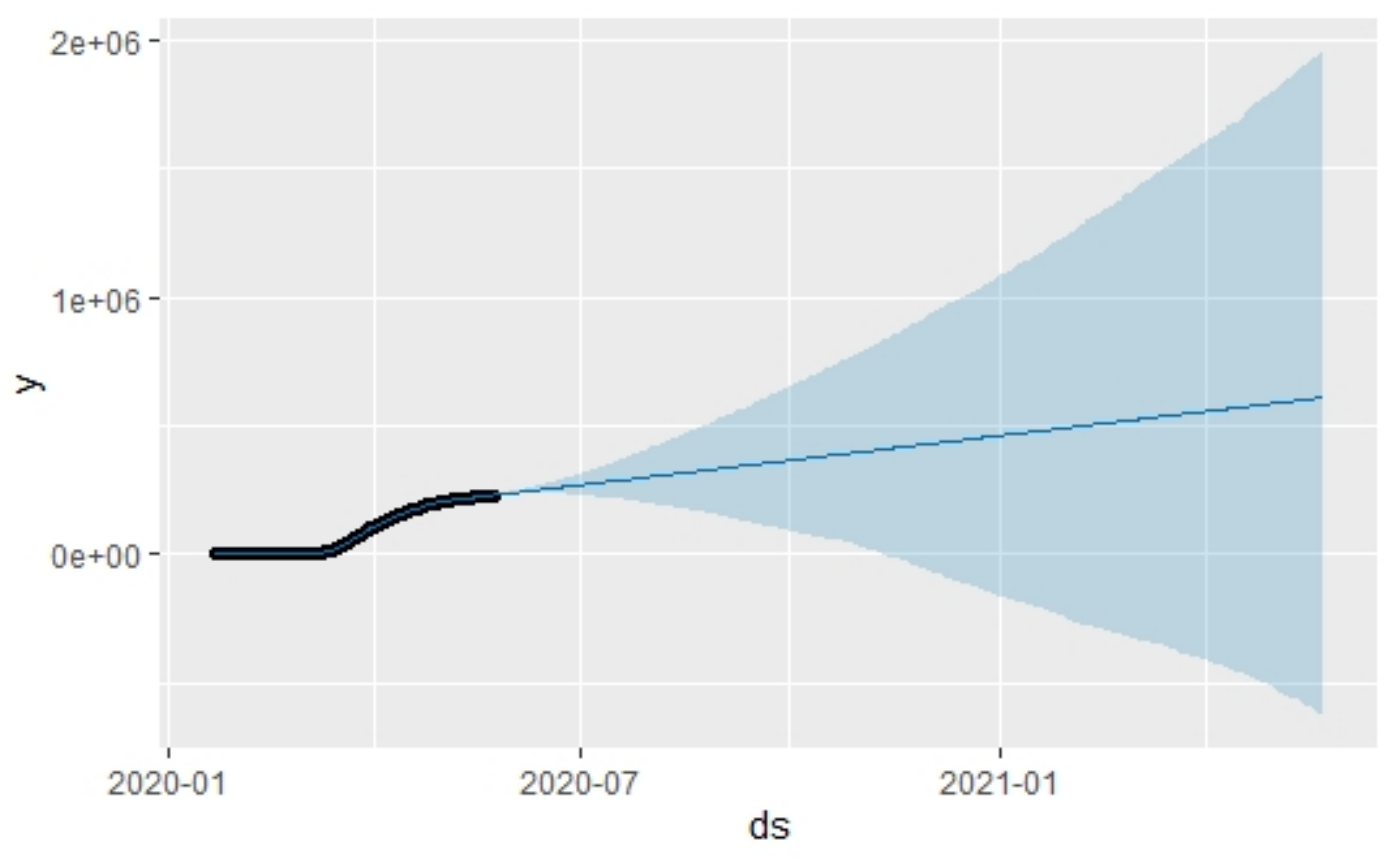

263

Figure 21 Forcasting by Prophet by the next year (Confirmed cases in Italy)

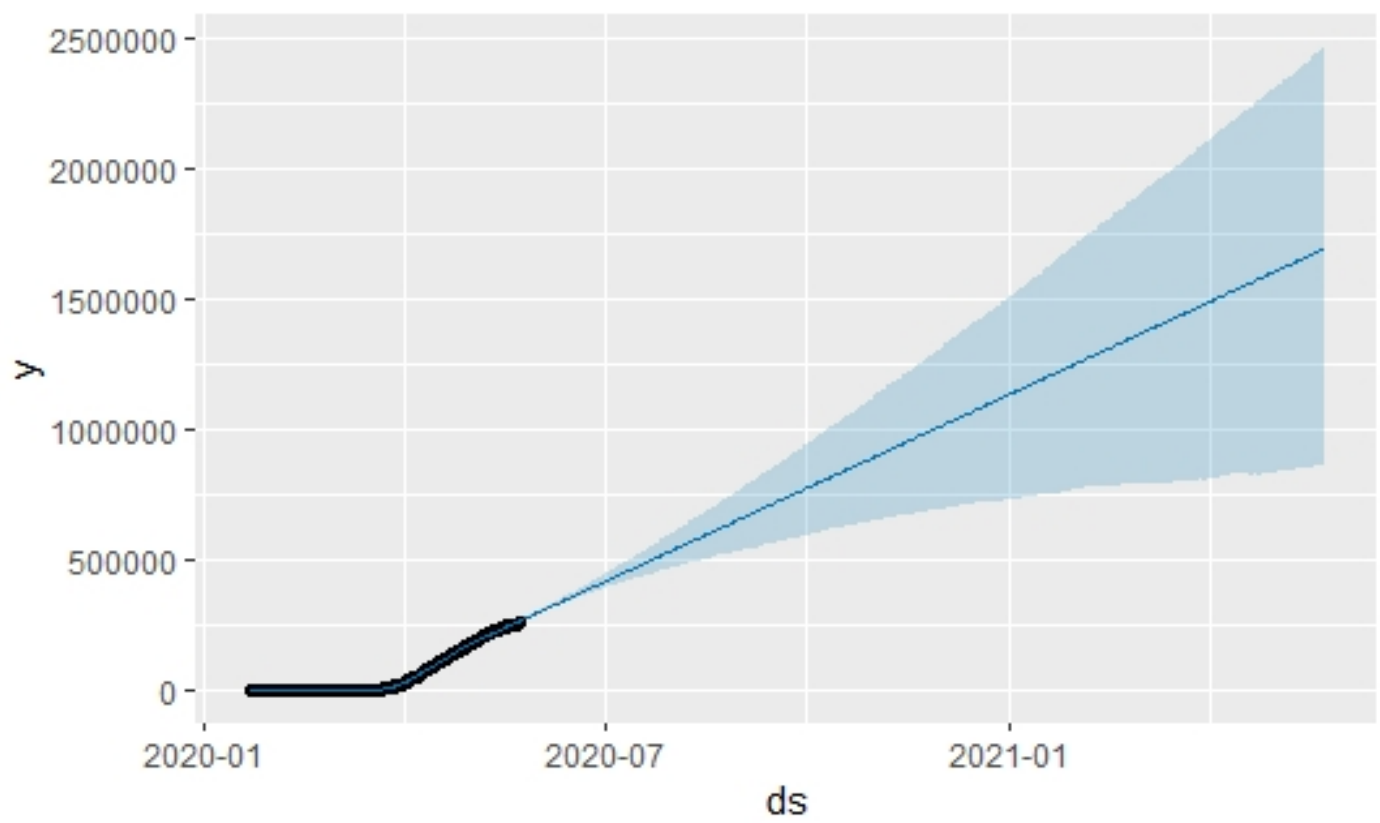




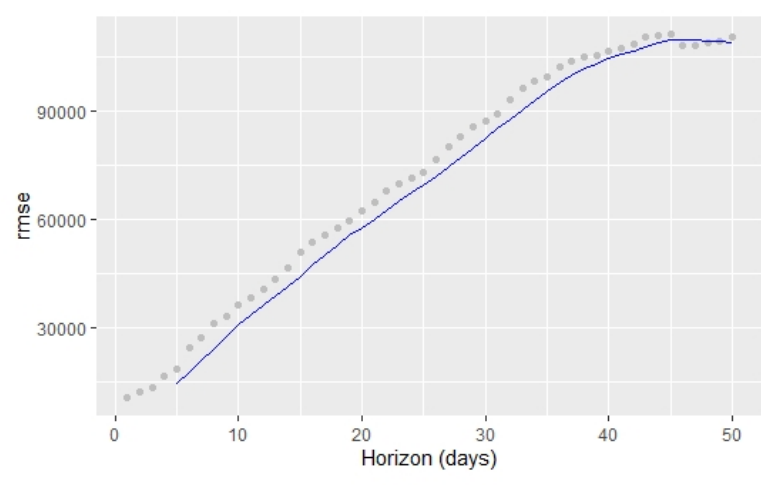

(a) United Kingdom

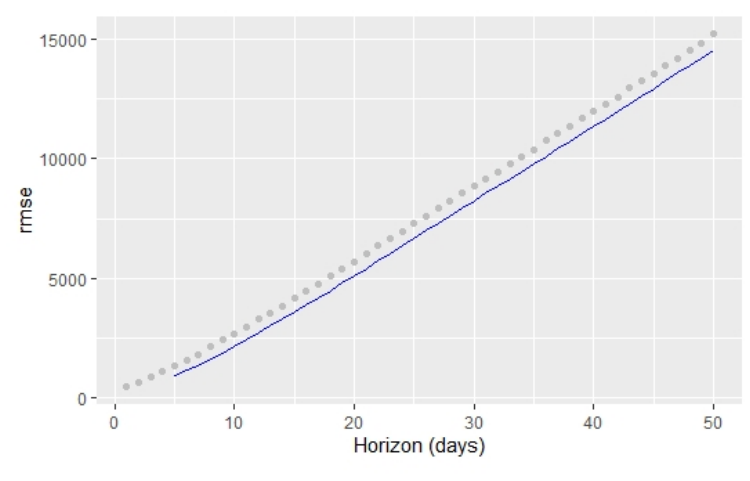

(b) Australia

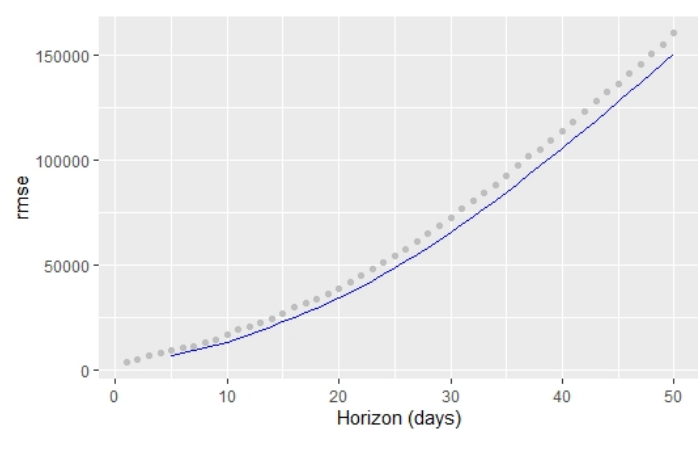

(C) Italy 
277 VI. Conclusion and discussion

COVID-19 is a family of Coronaviruses that has affected the life of billions of people worldwide. The first phase of the paper started with a short analysis of COVID-19, focusing on Australia, Italy, and the United Kingdom. The analysis presents confirmed and death growth rates in Australia, a comparison between Australia, Italy, and the United Kingdom, and also, a short analysis in different states of Australia. The analysis shows that generally Australia is in a good position compared with two other countries. However, the situation in different cities of Australia are completely complicated; for example, New South Wales has the most confirmed and deaths cases, while Northern Territory shows the least confirmed and death cases (it is valuable to mention that New South Wales has more population).

Mathematical approaches based on SIR and SEIR were proposed to predict the epidemiology in Australia, Italy, and the United Kingdom. Since the classic form of SIR and SEIR are deterministic, an improved version based on parameter optimization was suggested to improve the prediction. The results are compared with logistic function and Prophet algorithm and summarized as follows:

- Comparison between the classic form of SIR model with real data showed a significant gap. However, initializing the parameters of the SIR model significantly improved the prediction.

- The classic form of SIR model worked better for the United Kingdom, while the SIR model was not suitable for Australia case (regarding RMSE values).

- The logistic function was a good model for the United Kingdom with an r2_score of 0.97 , while this score for Australia was 0.67 and Italy was 0.95 .

- The best RMSE value belonged to the Australia cases (confirmed and deaths).

- Optimization of parameters of the SIR and SEIR models significantly improved the prediction accuracy of the models. 
- Improved version of SEIR has better performance compared with SIR model (Regarding RMSE values and Figures).

- Optimized SEIR model has better prediction for UK and Italy compared with Australia.

- The best values for the parameters were found using the Nelder-Mead algorithm for SIR model and L-BFGS-B algorithm for SEIR model.

- The Prophet algorithm worked better for Italy and the United Kingdom cases than for Australian cases. logistic function, Prophet algorithm, and classic form of SIR model.

In this paper, all forecasting was addressed without considering of scenario of social distancing and quarantine that makes it valuable as a future direction. This paper presents SIR and SEIR as epidemiology models; it would interesting to test other epidemiology models. Moreover, it is worthwhile to combine the mathematical model with other observations such as Policy intervention, human behavior, and constraints.

323

324

References 
Arkes, H. R. (2001). Overconfidence in judgmental forecasting. Principles of forecasting, Springer: 495515.

Armstrong, J. S. (2001). Standards and practices for forecasting. Principles of Forecasting, Springer: 679732.

Byrd, R. H., P. Lu, J. Nocedal and C. Zhu (1995). "A limited memory algorithm for bound constrained optimization." SIAM Journal on scientific computing 16(5): 1190-1208.

Capasso, V. and G. Serio (1978). "A generalization of the Kermack-McKendrick deterministic epidemic model." Mathematical Biosciences 42(1-2): 43-61.

Fletcher, R. (1987). "Practical methods of optimization. 1987." John and Sons, Chichester.

Fletcher, R. and C. M. Reeves (1964). "Function minimization by conjugate gradients." The computer journal 7(2): 149-154.

Kermack, W. O. and A. G. McKendrick (1932). "Contributions to the mathematical theory of epidemics. II.-The problem of endemicity." Proceedings of the Royal Society of London. Series A, containing papers of a mathematical and physical character 138(834): 55-83.

Maleki, M., M. R. Mahmoudi, D. Wraith, K.-H. J. T. M. Pho and I. Disease (2020). "Time series modelling to forecast the confirmed and recovered cases of COVID-19." 101742.

Mbuvha, R. R. and T. Marwala (2020). "On Data-Driven Management of the COVID-19 Outbreak in South Africa." medRxiv. Mitchell, T. M. (1997). Machine learning, McGraw-hill New York.

Ndiaye, B. M., L. Tendeng and D. Seck (2020). "Analysis of the COVID-19 pandemic by SIR model and machine learning technics for forecasting." arXiv preprint arXiv:2004.01574.

Nelder, J. A. and R. Mead (1965). "A simplex method for function minimization." The computer journal 7(4): 308-313.

Nikolopoulos, K., A. Litsa, F. Petropoulos, V. Bougioukos and M. Khammash (2015). "Relative performance of methods for forecasting special events." Journal of Business Research 68(8): 1785-1791. Peng, L., W. Yang, D. Zhang, C. Zhuge and L. Hong (2020). "Epidemic analysis of COVID-19 in China by dynamical modeling." arXiv preprint arXiv:2002.06563.

Putra, S. and Z. Khozin Mu'tamar (2019). "Estimation of Parameters in the SIR Epidemic Model Using Particle Swarm Optimization." American Journal of Mathematical and Computer Modelling 4(4): 83-93. Qi, H., S. Xiao, R. Shi, M. P. Ward, Y. Chen, W. Tu, Q. Su, W. Wang, X. Wang and Z. J. S. o. t. T. E. Zhang (2020). "COVID-19 transmission in Mainland China is associated with temperature and humidity: A timeseries analysis." 138778.

Salgotra, R., M. Gandomi and A. H. Gandomi (2020). "Time Series Analysis and Forecast of the COVID-19 Pandemic in India using Genetic Programming." Chaos, Solitons \& Fractals: 109945.

Taylor, S. and B. Letham (2017). "prophet: Automatic forecasting procedure." R package version 0.21.

Taylor, S. J. and B. Letham (2018). "Forecasting at scale." The American Statistician 72(1): 37-45. Weiss, H. H. (2013). "The SIR model and the foundations of public health." Materials matematics: 00010017. 


\section{Figures}

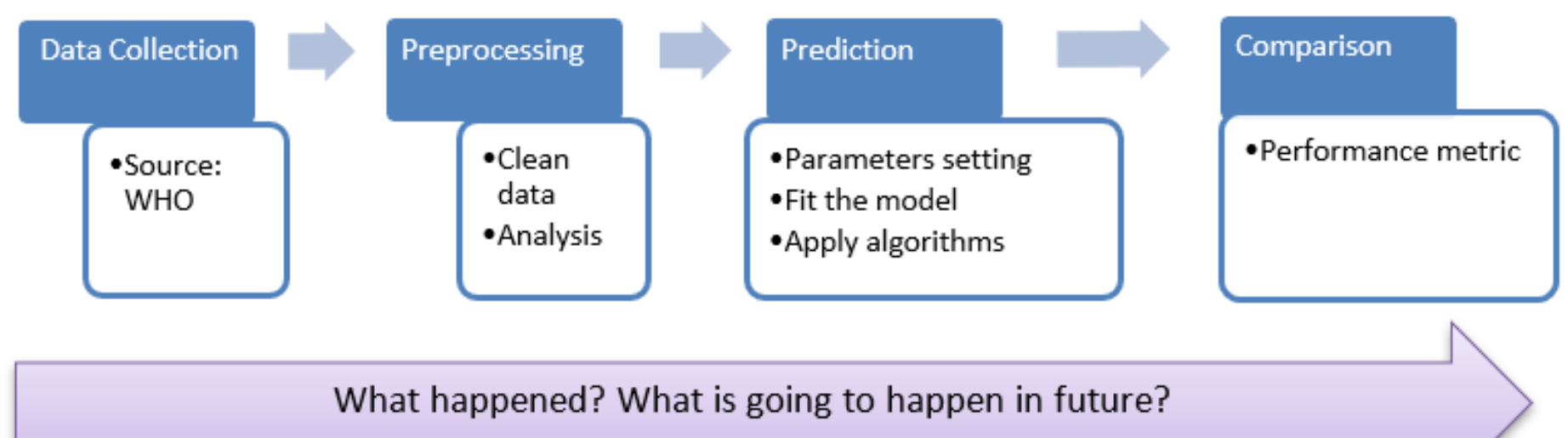

\section{Figure 1}

Flowchart of the current research process

\section{Susceptible (S)}

\section{Infectious (I)}

\section{Recovered (R)}

\section{Figure 2}

SIR model

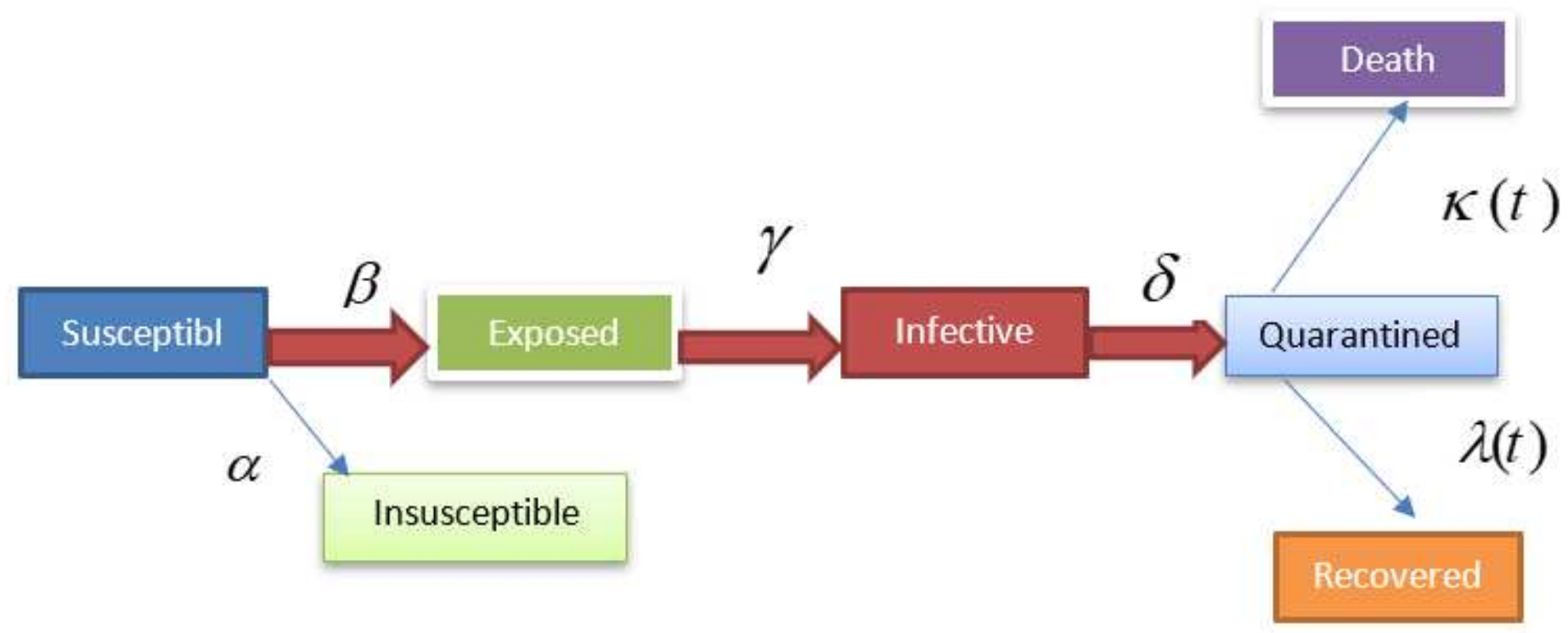

Figure 3 
The SEIR diagram (Peng, Yang et al. 2020)

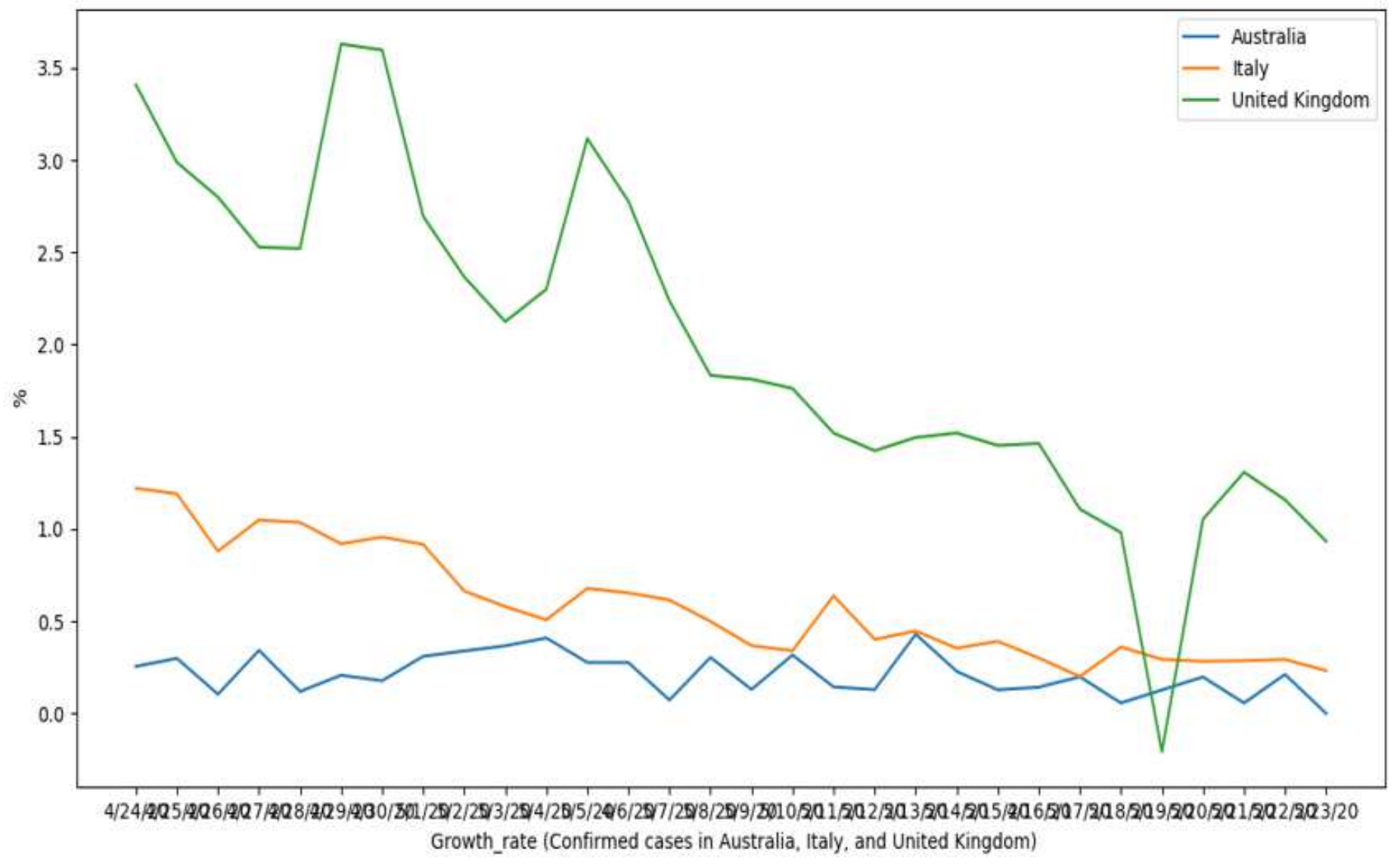

Figure 4

Growth rate (Confirmed cases in Australia, Italy, and the United Kingdom) 


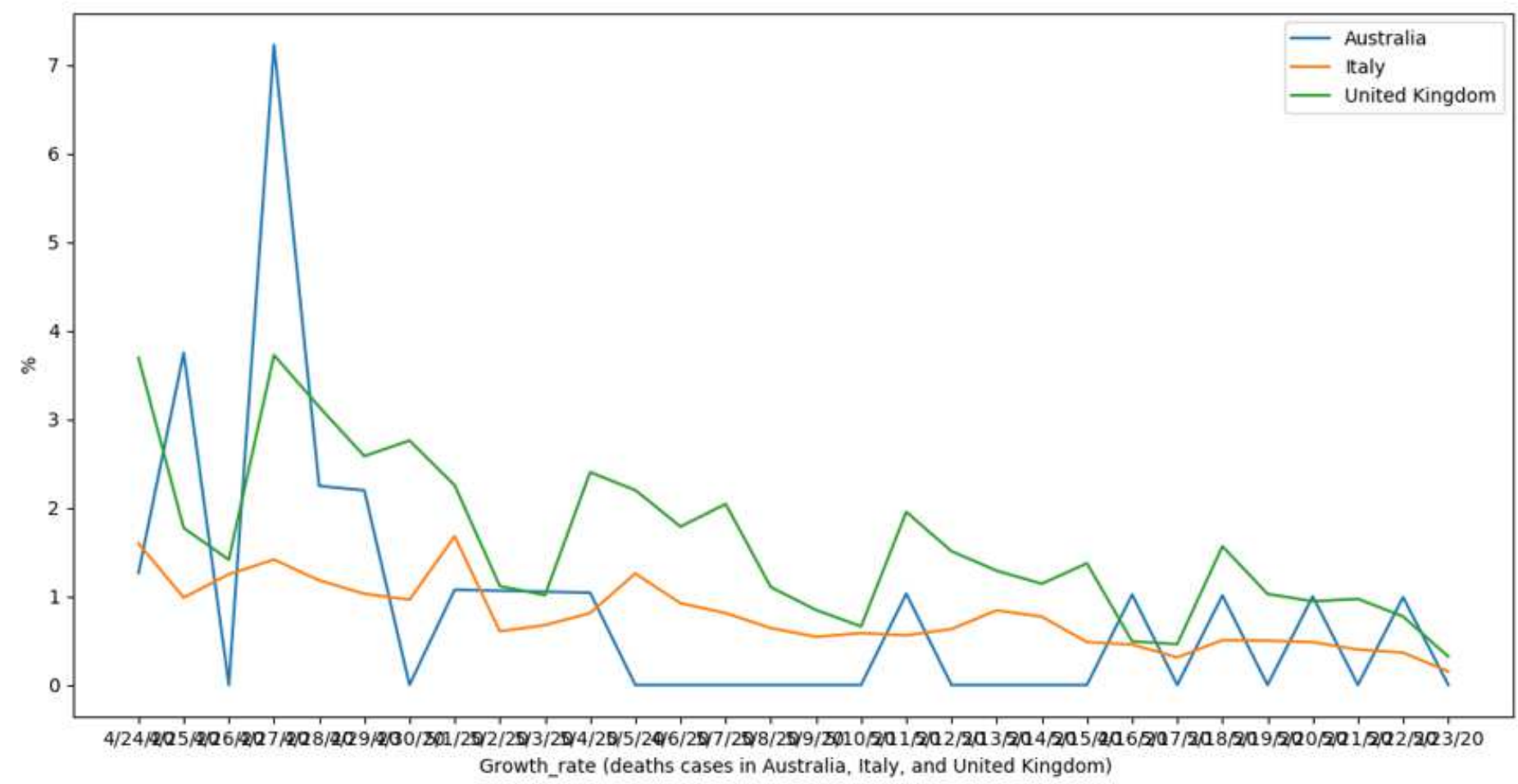

Figure 5

Growth rate (death cases in Australia, Italy, and the United Kingdom)

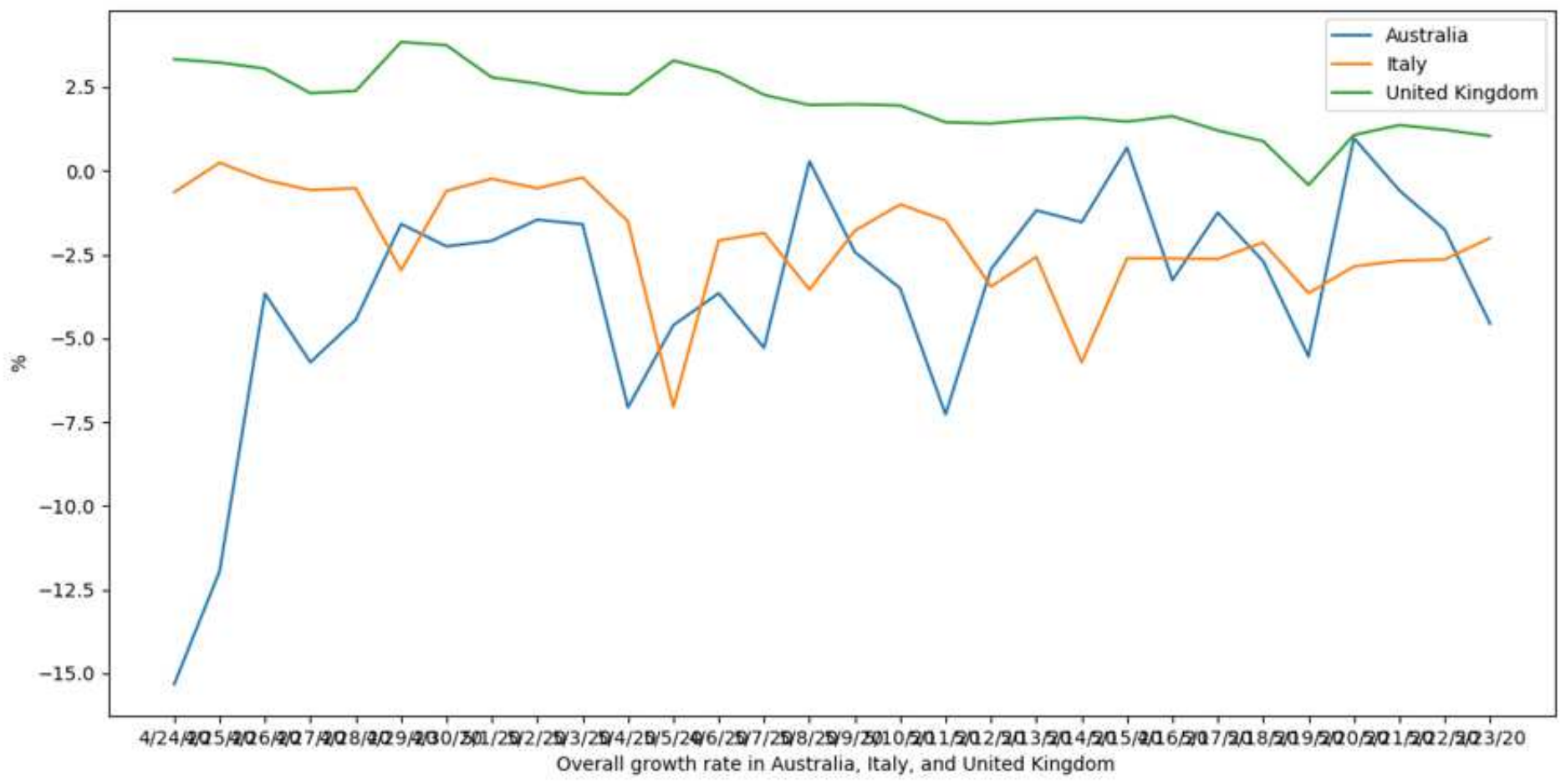

Figure 6 
Overall growth rate for confirmed cases in Australia, Italy, and the United Kingdom

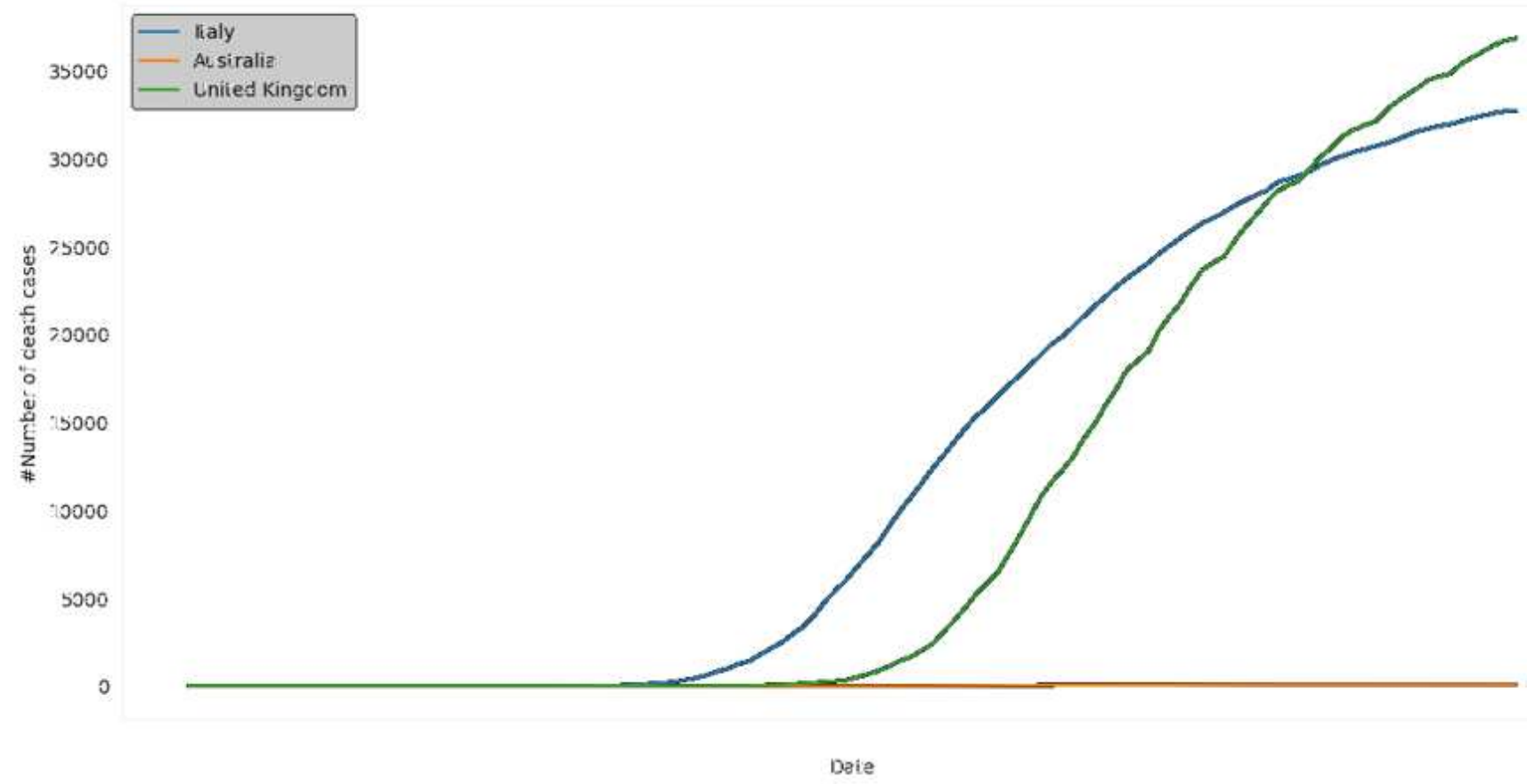

Figure 7

Number of death cases in Australia compared with Italy and the United Kingdom 


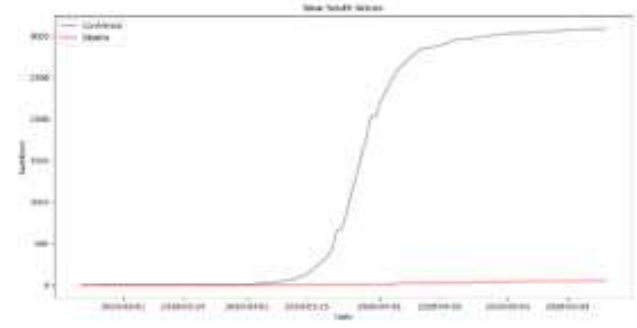

Figure $8(a)$ New South Wales

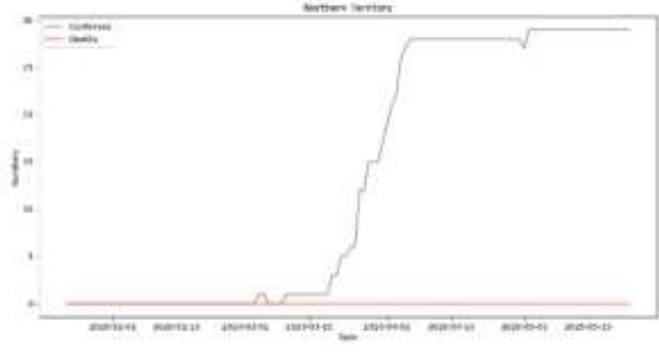

Figure 8(c) Northern Territory

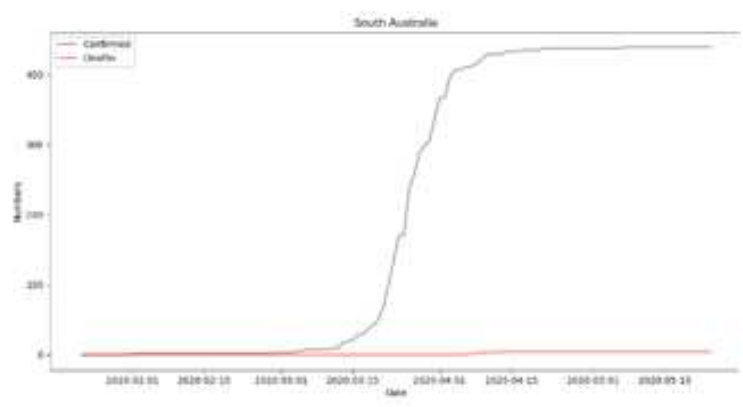

Figure 8(e) South Australia

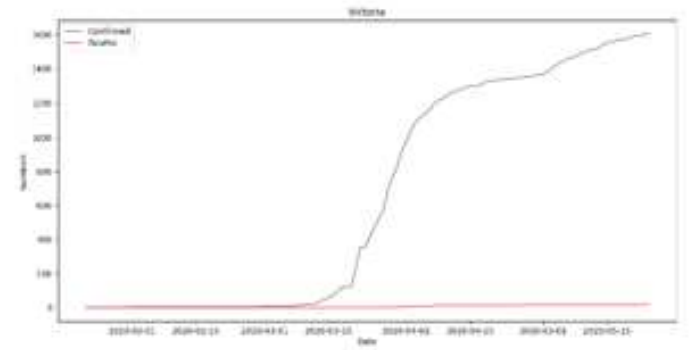

Figure $8(g)$ Victoria

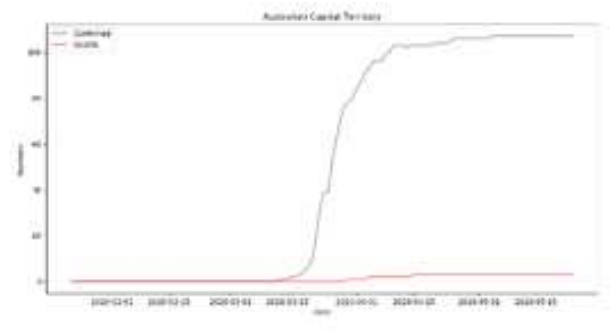

Figure 8 (b) Australian Capital Territory

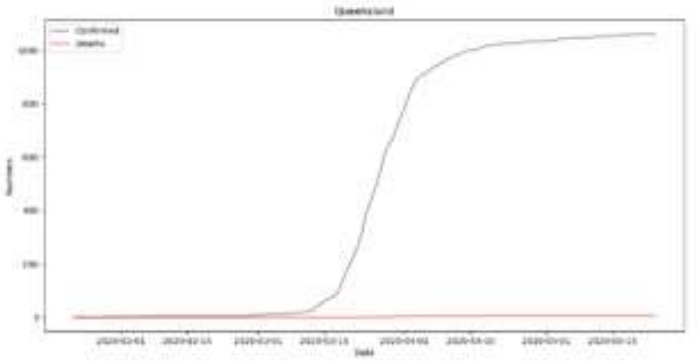

Figure 8(d) Queensland

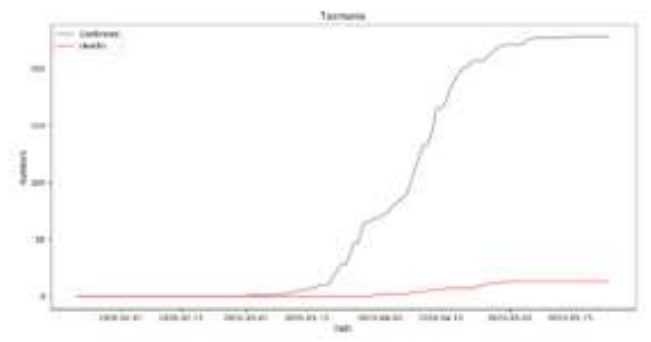

Figure 8(f) Tasmania

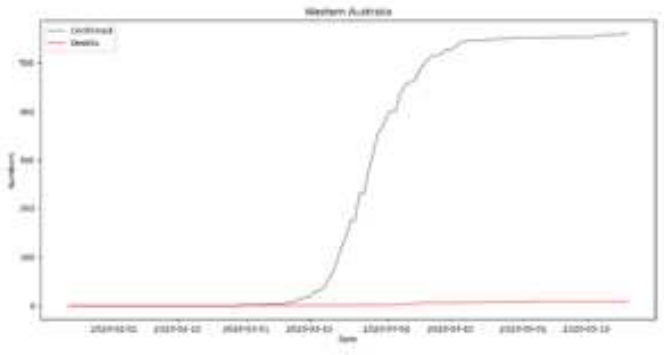

Figure 8(h) Western Australia

\section{Figure 8}

Confirmed versus death cases in different Australian states 
Prediction of confirmed cases in Australia by logistic function

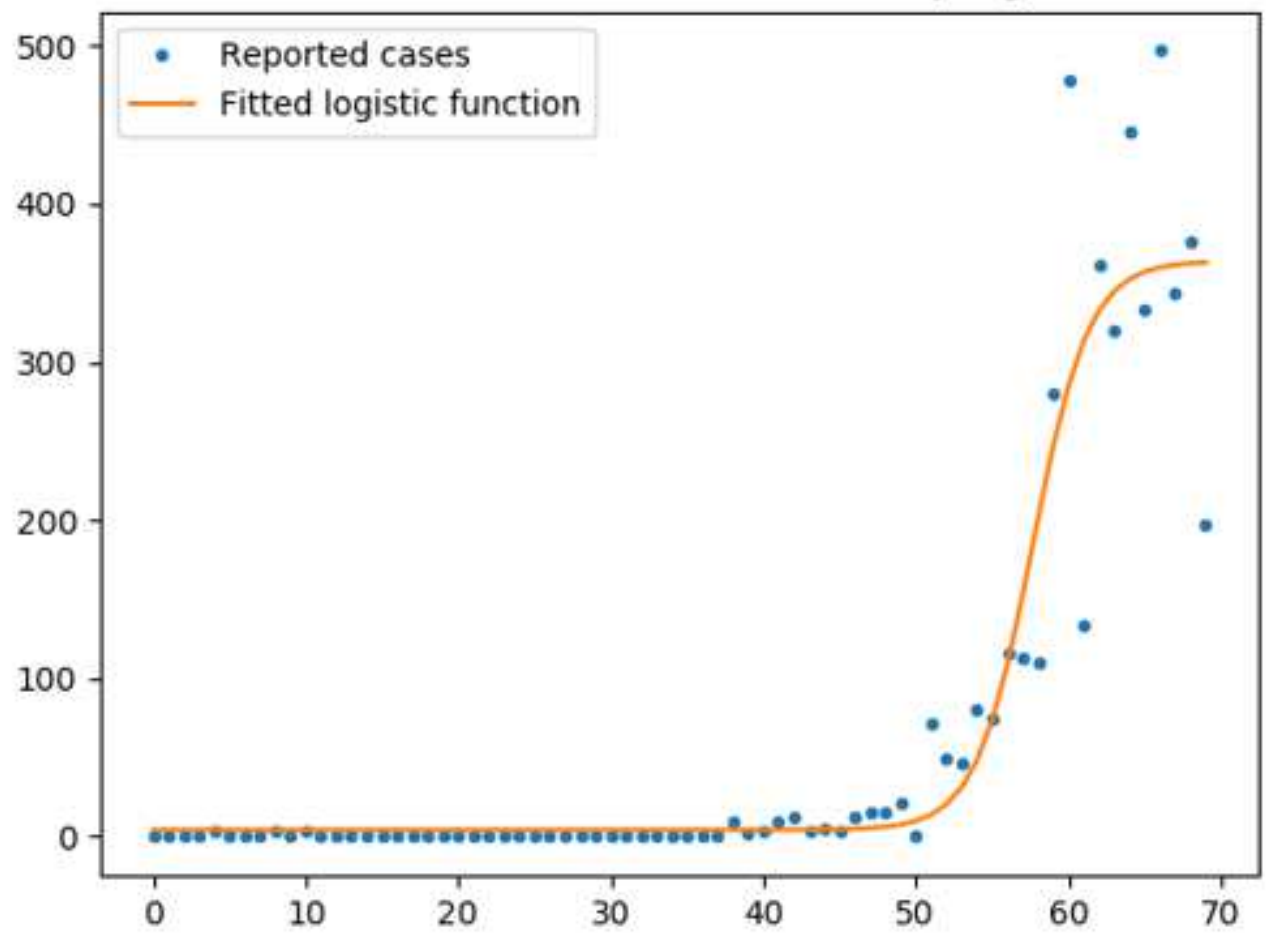

Figure 9

Prediction of confirmed cases by logstic function (Australia)

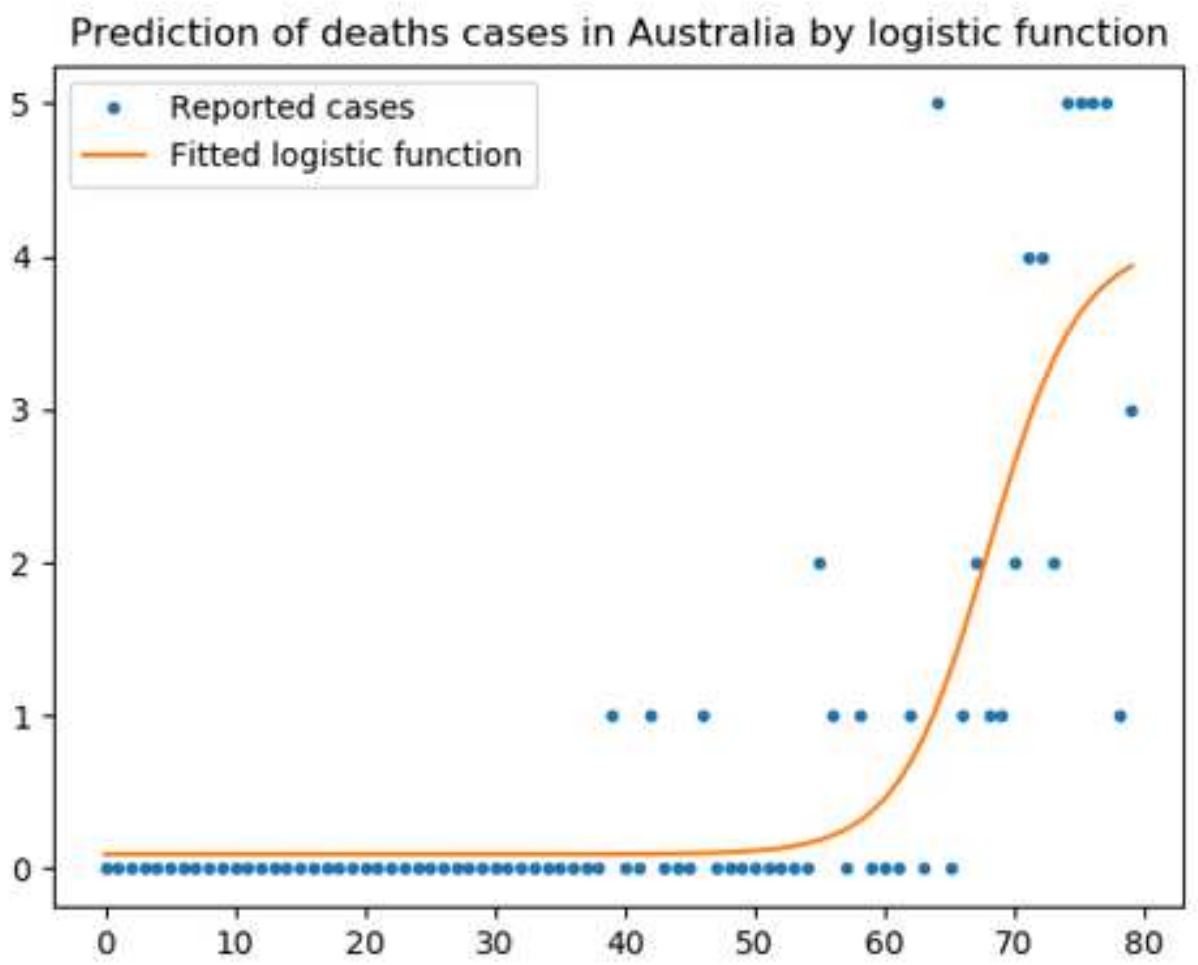

Figure 10 
Prediction of death cases by logistic function (Australia)

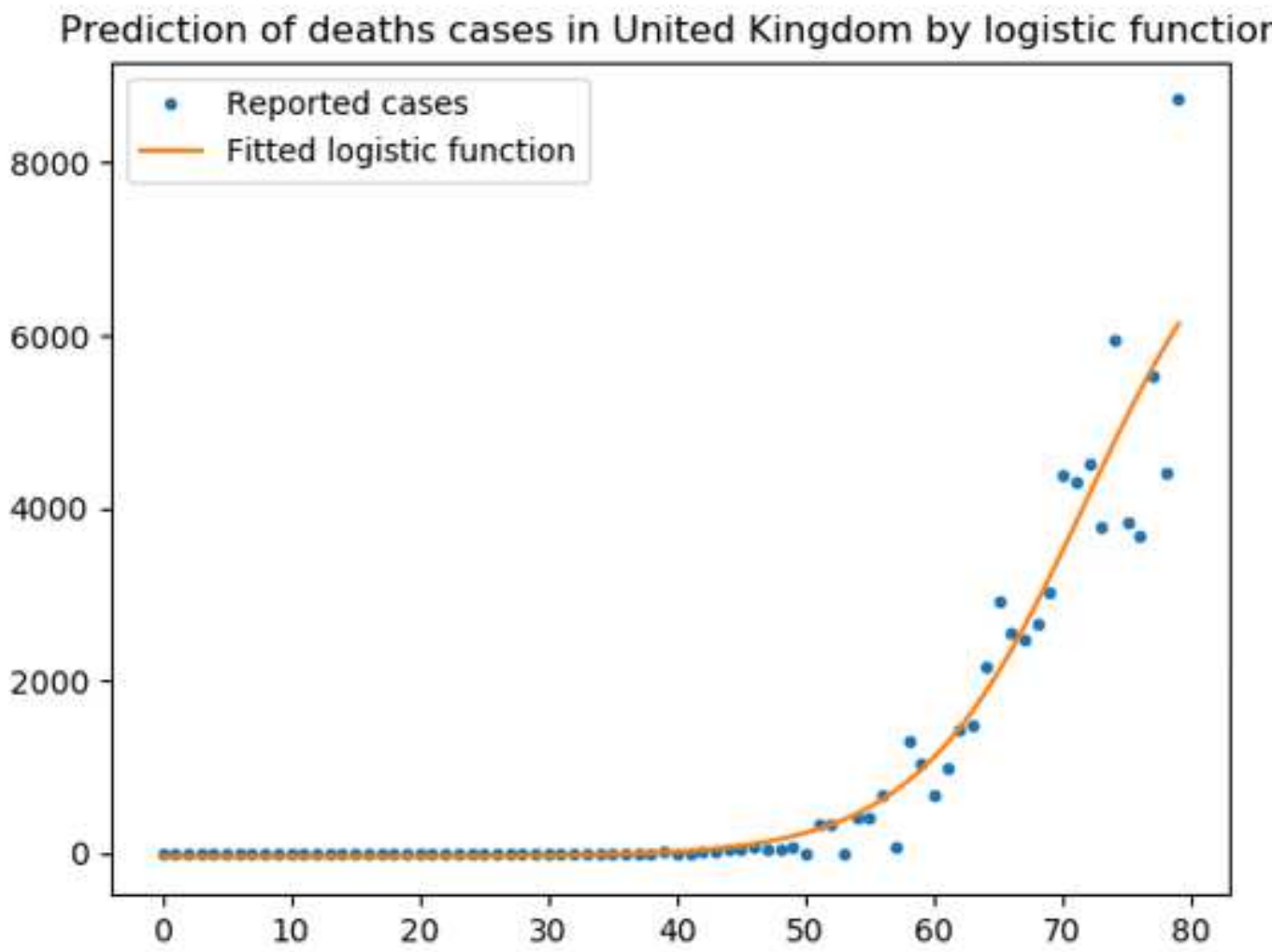

Figure 11

Prediction of confirmed cases by logstic function (United Kingdom) 
Prediction of deaths cases in United Kingdom by logistic function

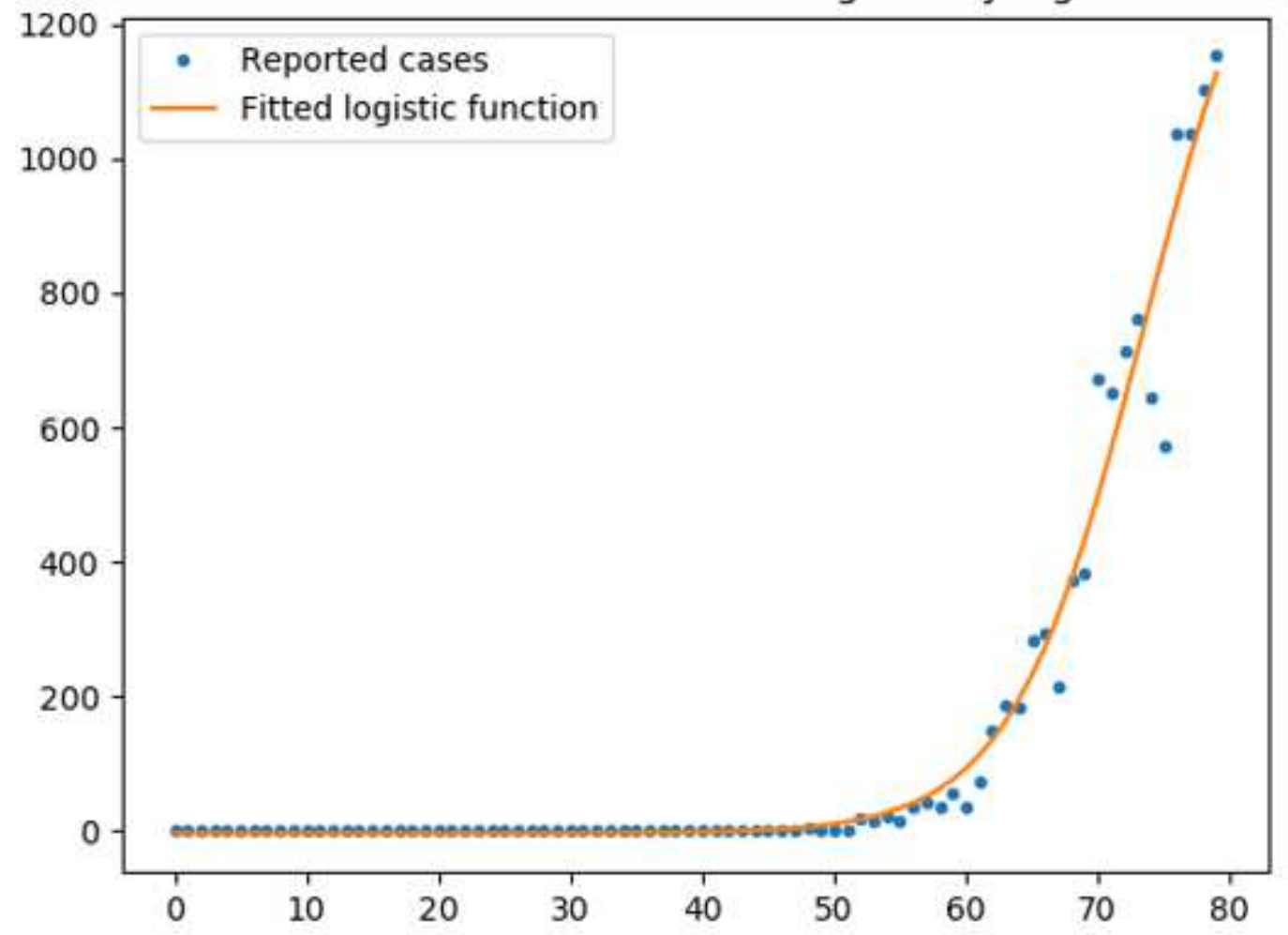

Figure 12

Prediction of deaths cases by logstic function (United Kingdom) 


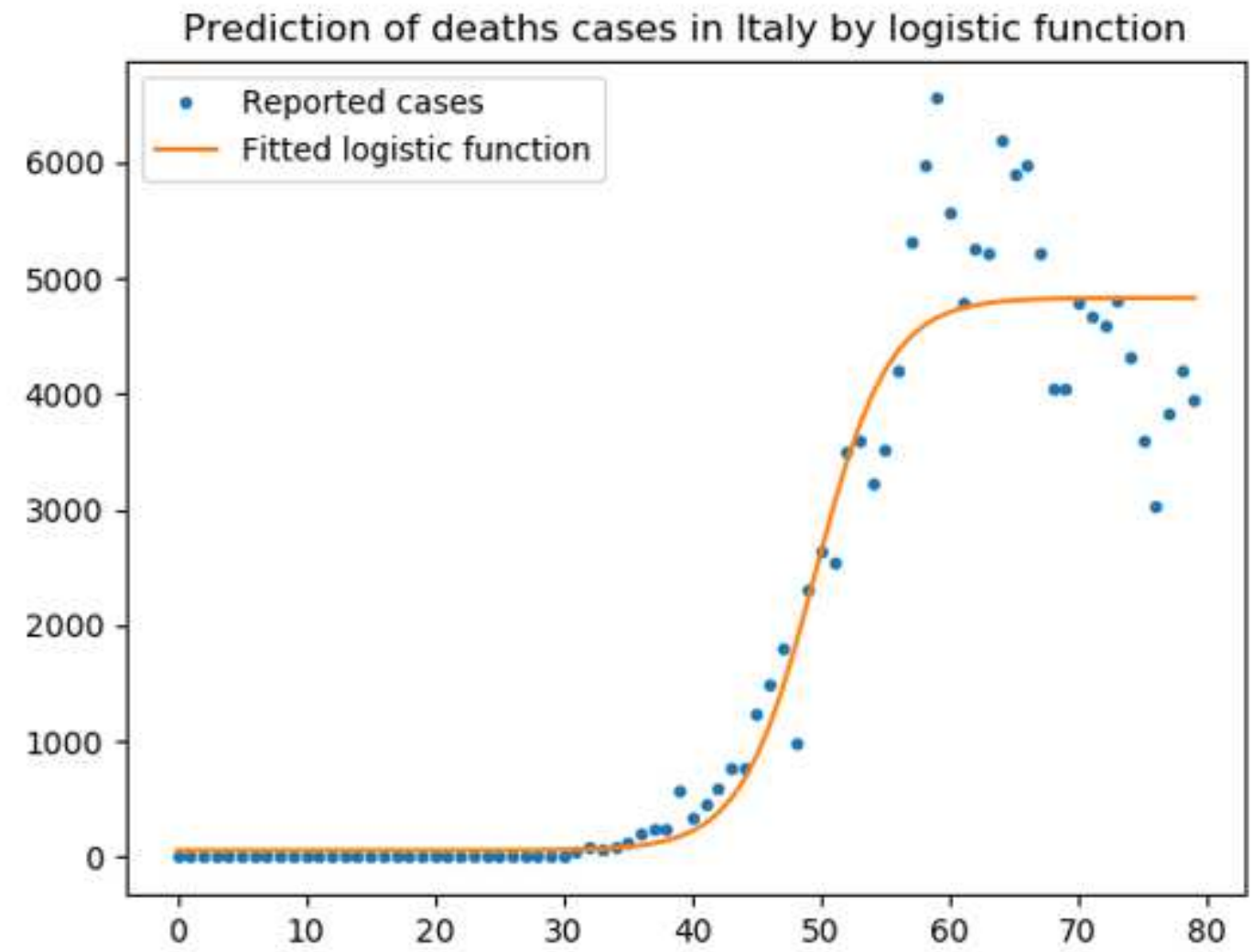

Figure 13

Prediction of confirmed cases by logstic function (Italy) 


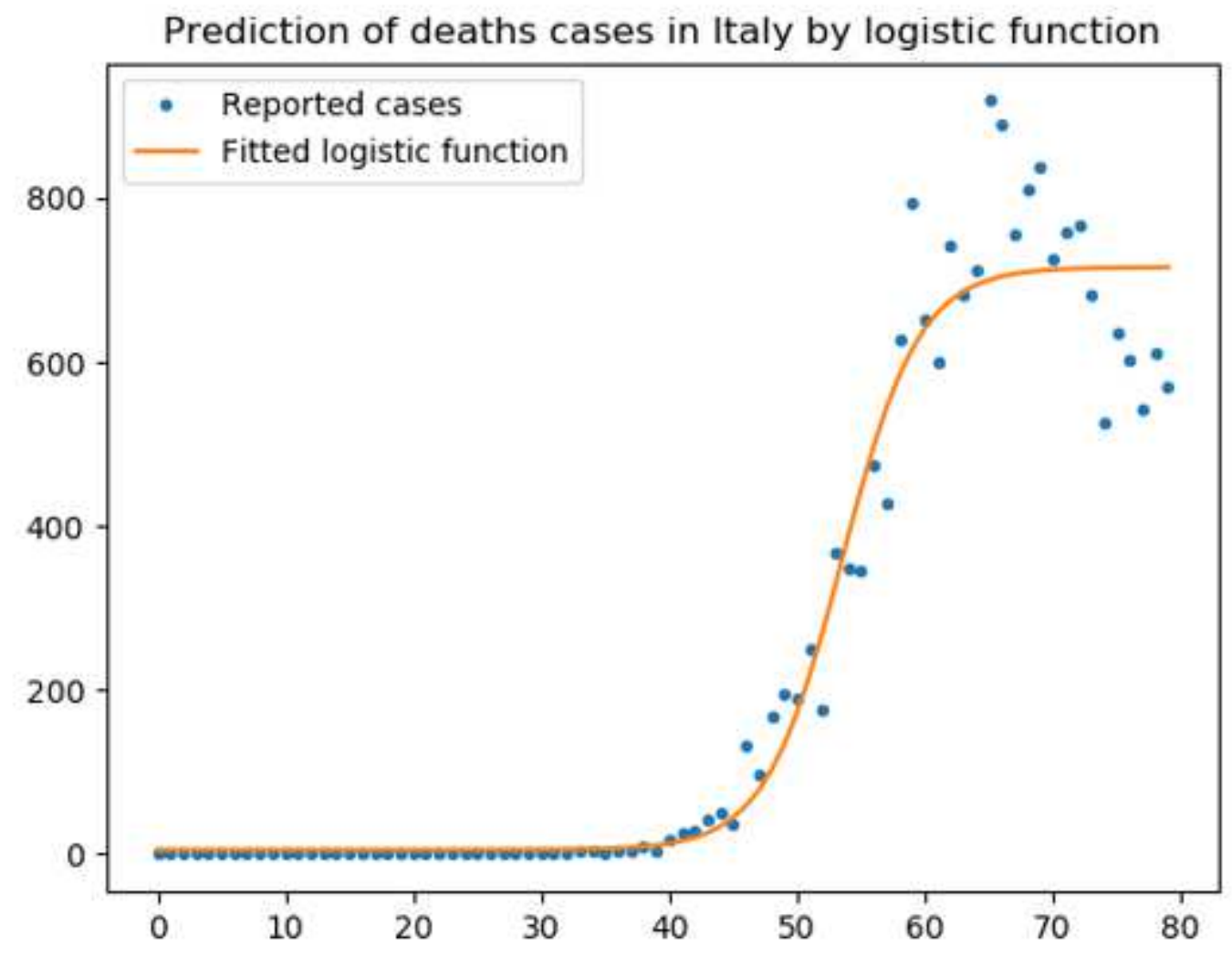

Figure 14

Prediction of deaths cases by logstic function (Italy)

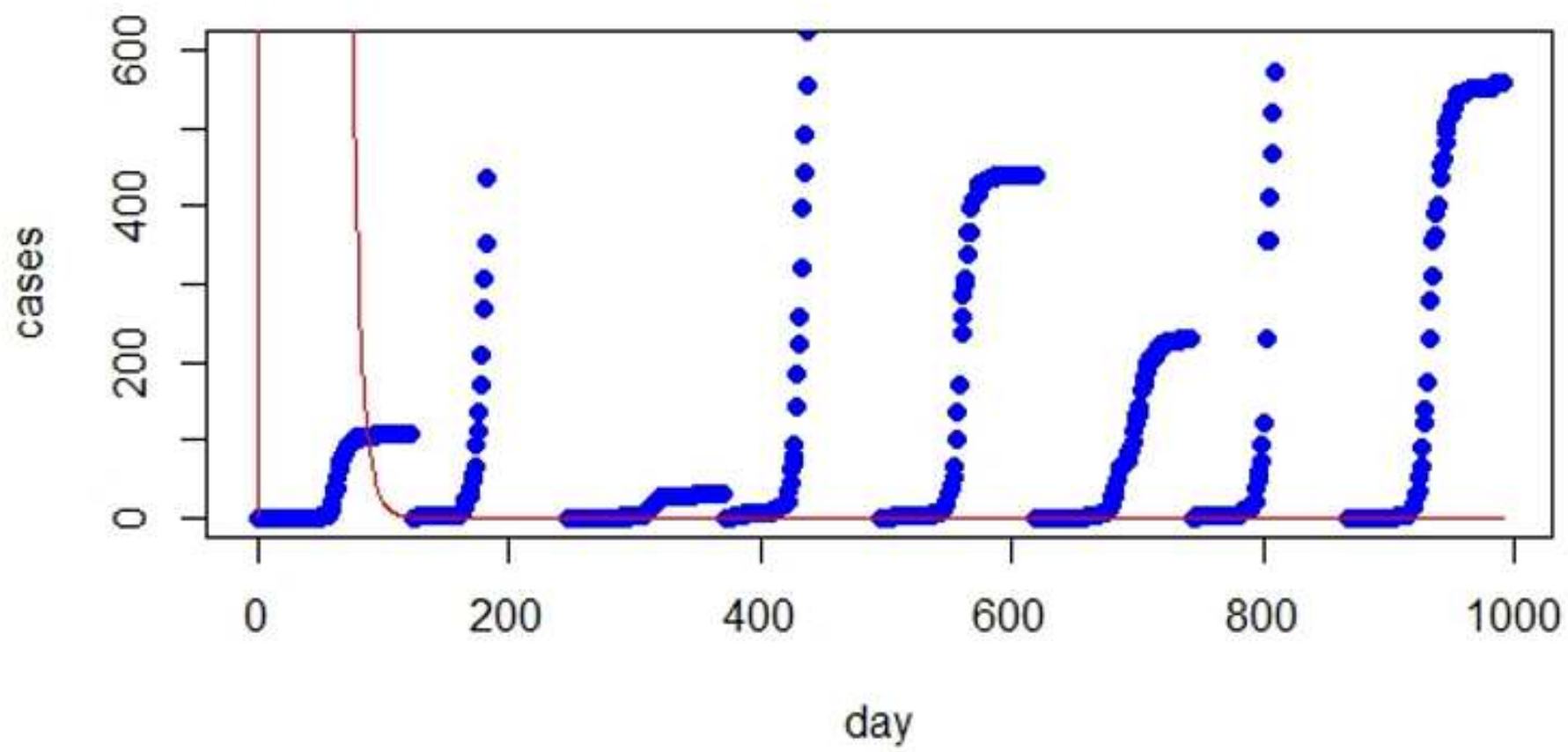

Figure 15 
Predicted cases in Australia using the susceptible, infected, recovered (SIR) model (blue: real confirmed cases, red: SIR model)

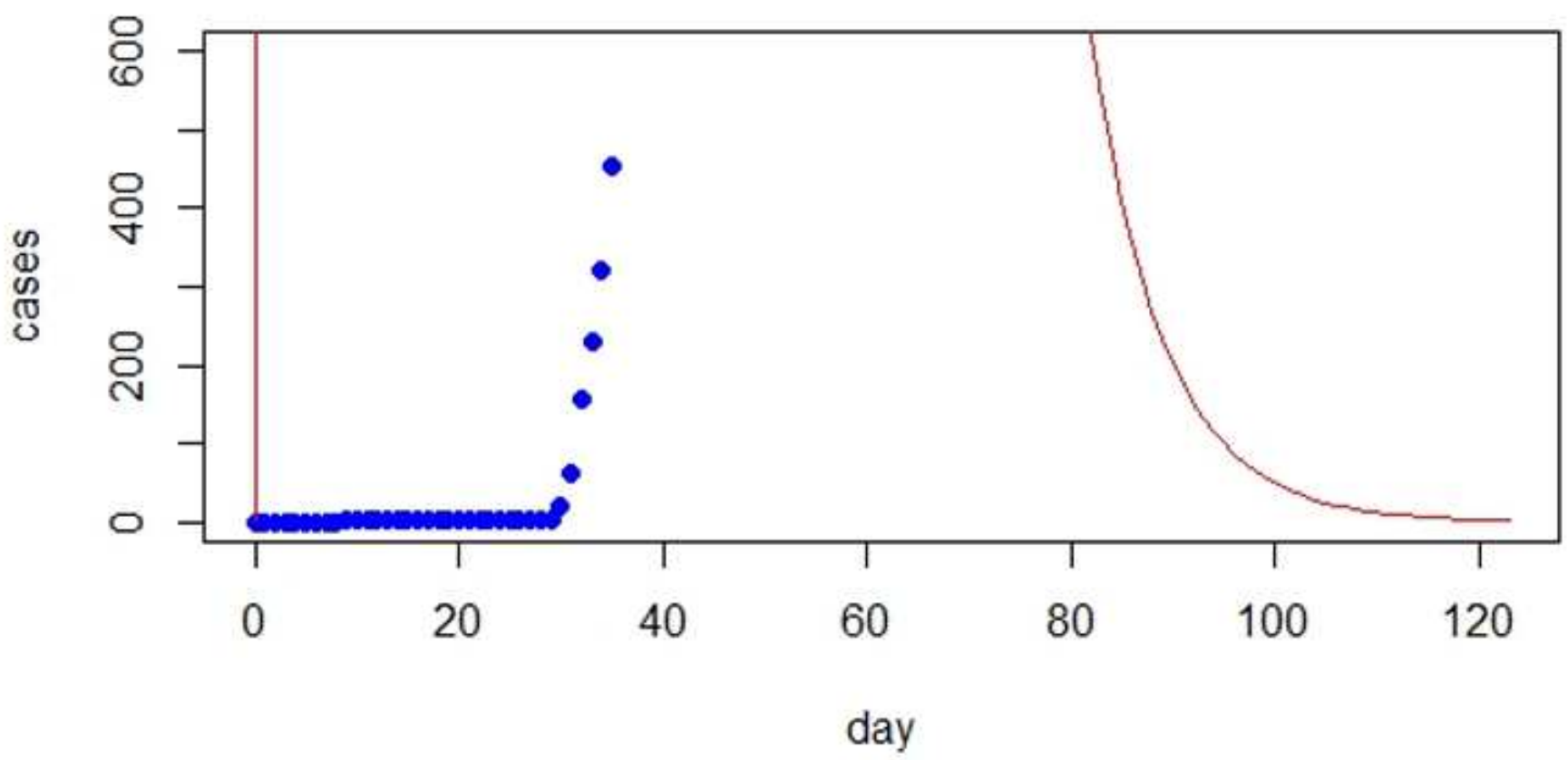

Figure 16

Predicted cases in Italy based on the SIR model (blue: real confirmed cases, red: SIR model)

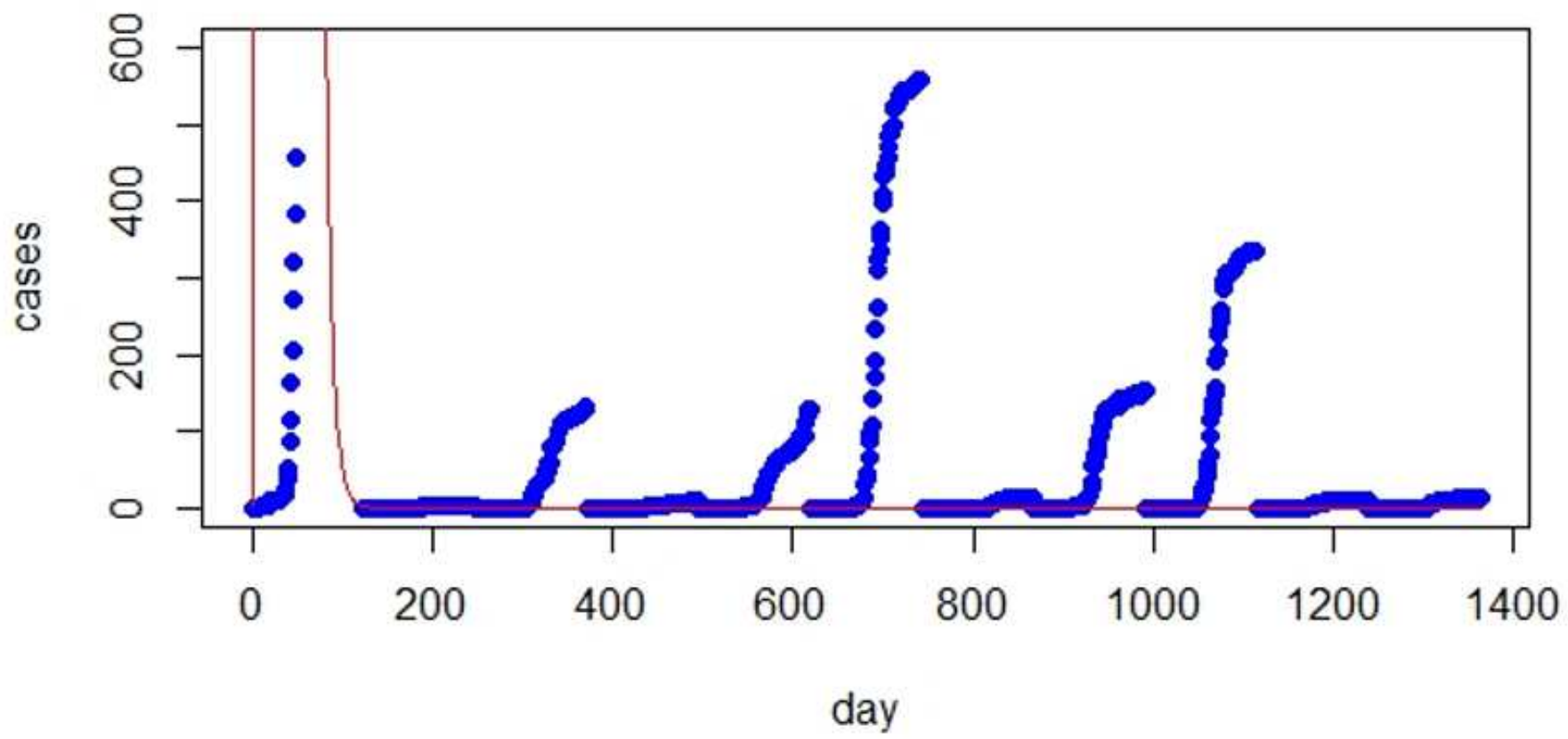

Figure 17 
Predicted cases in UK based on the SIR model (blue: real confirmed cases, red: SIR model)
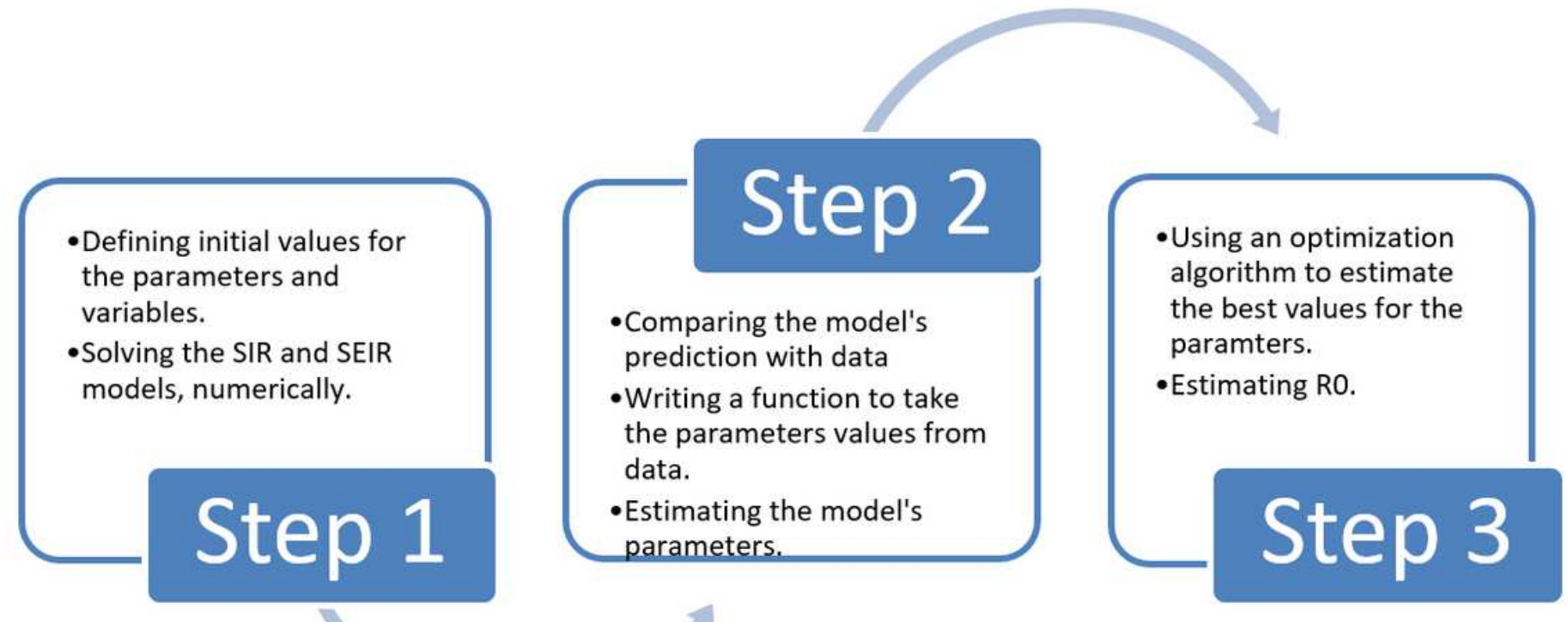

Figure 18

Flowchart of improved version of SIR and SEIR models

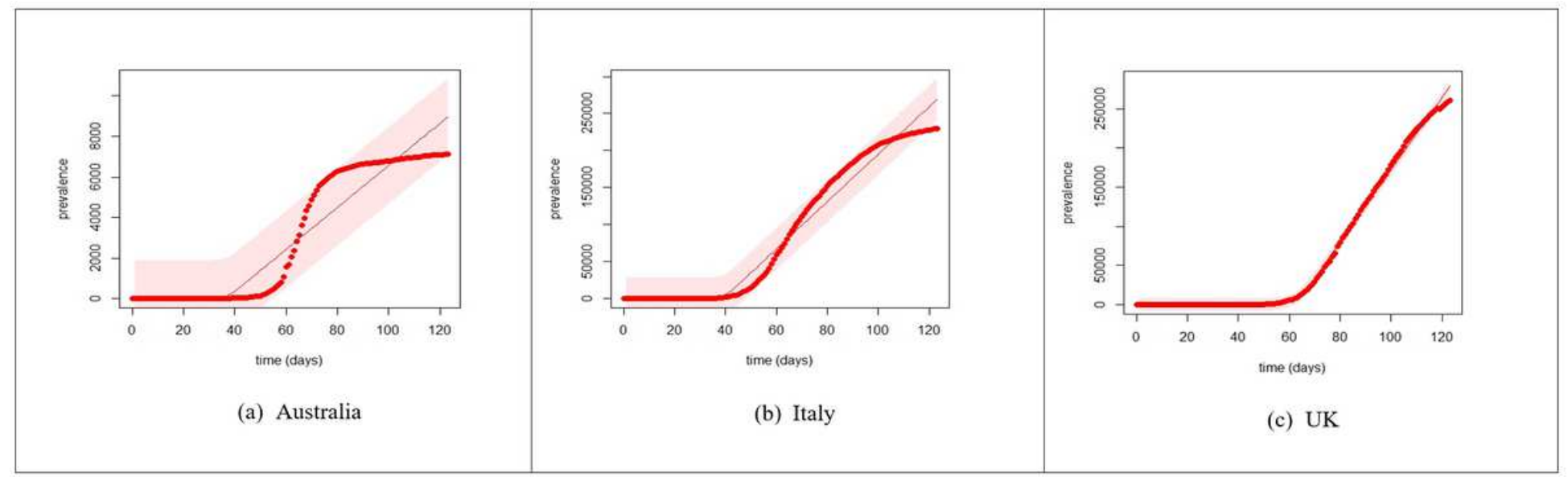

\section{Figure 19}

Prediction done by optimized SEIR model 


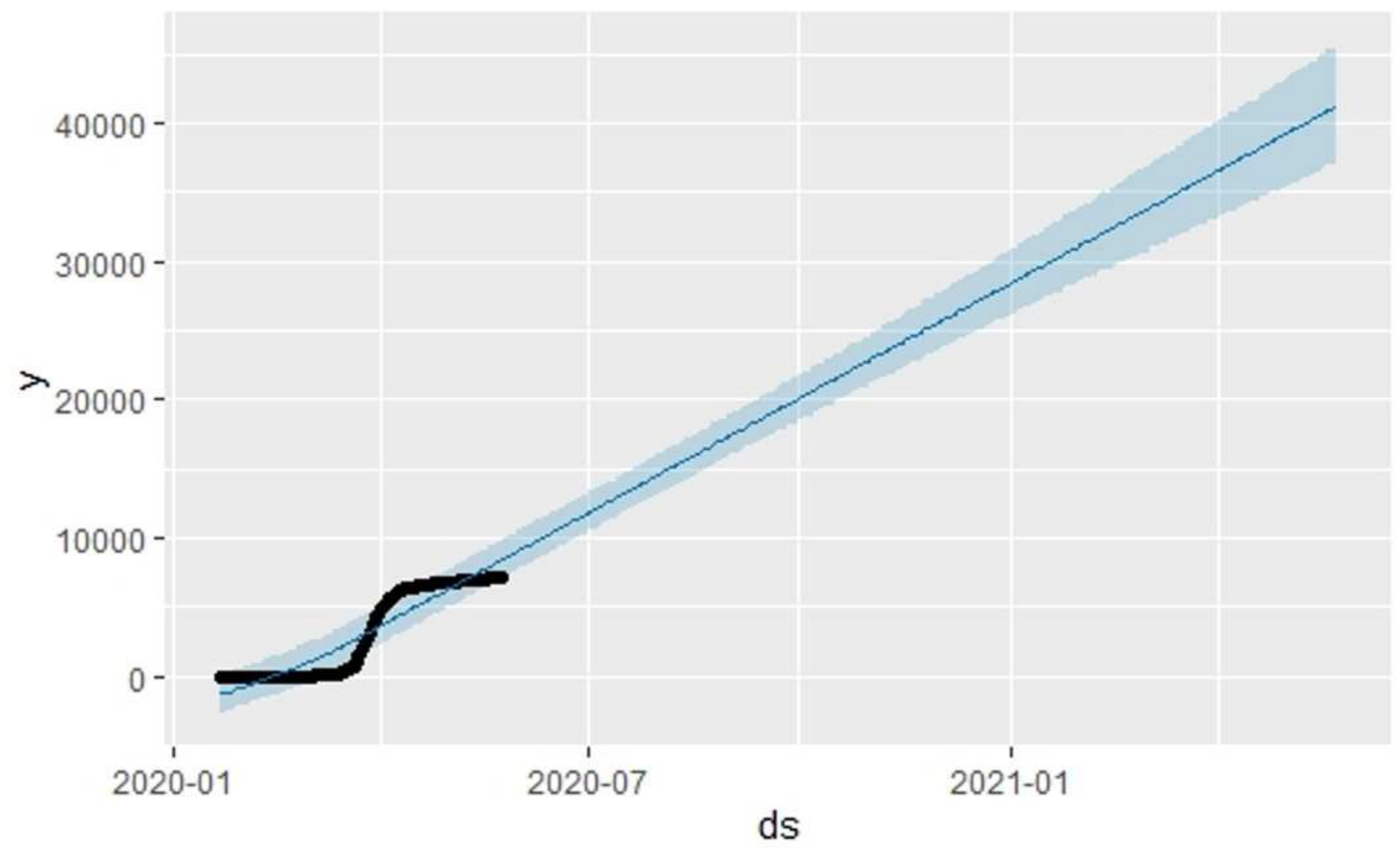

Figure 20

Forcasting by Prophet for the next year (Confirmed cases in Australia) 


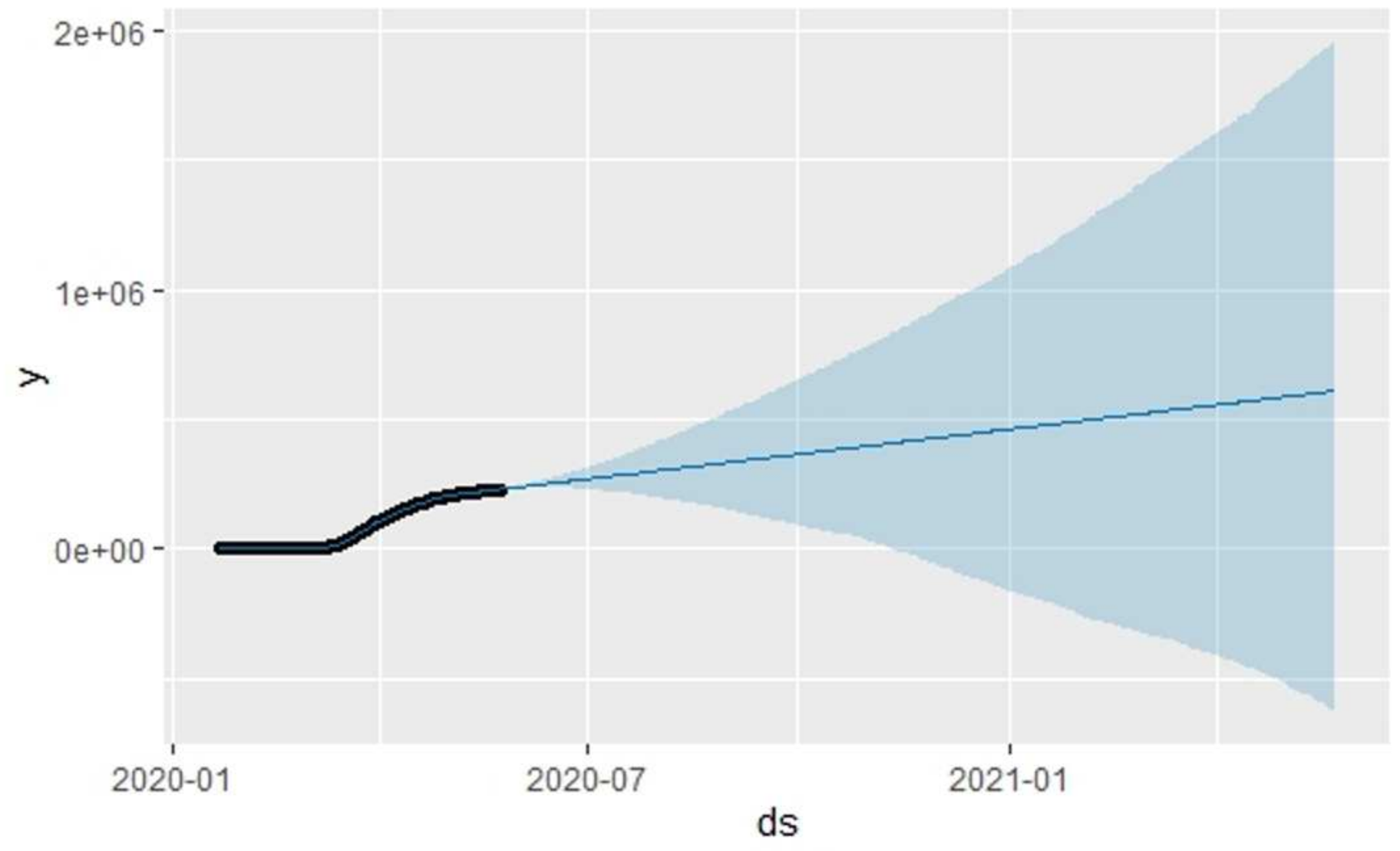

Figure 21

Forcasting by Prophet by the next year (Confirmed cases in Italy) 


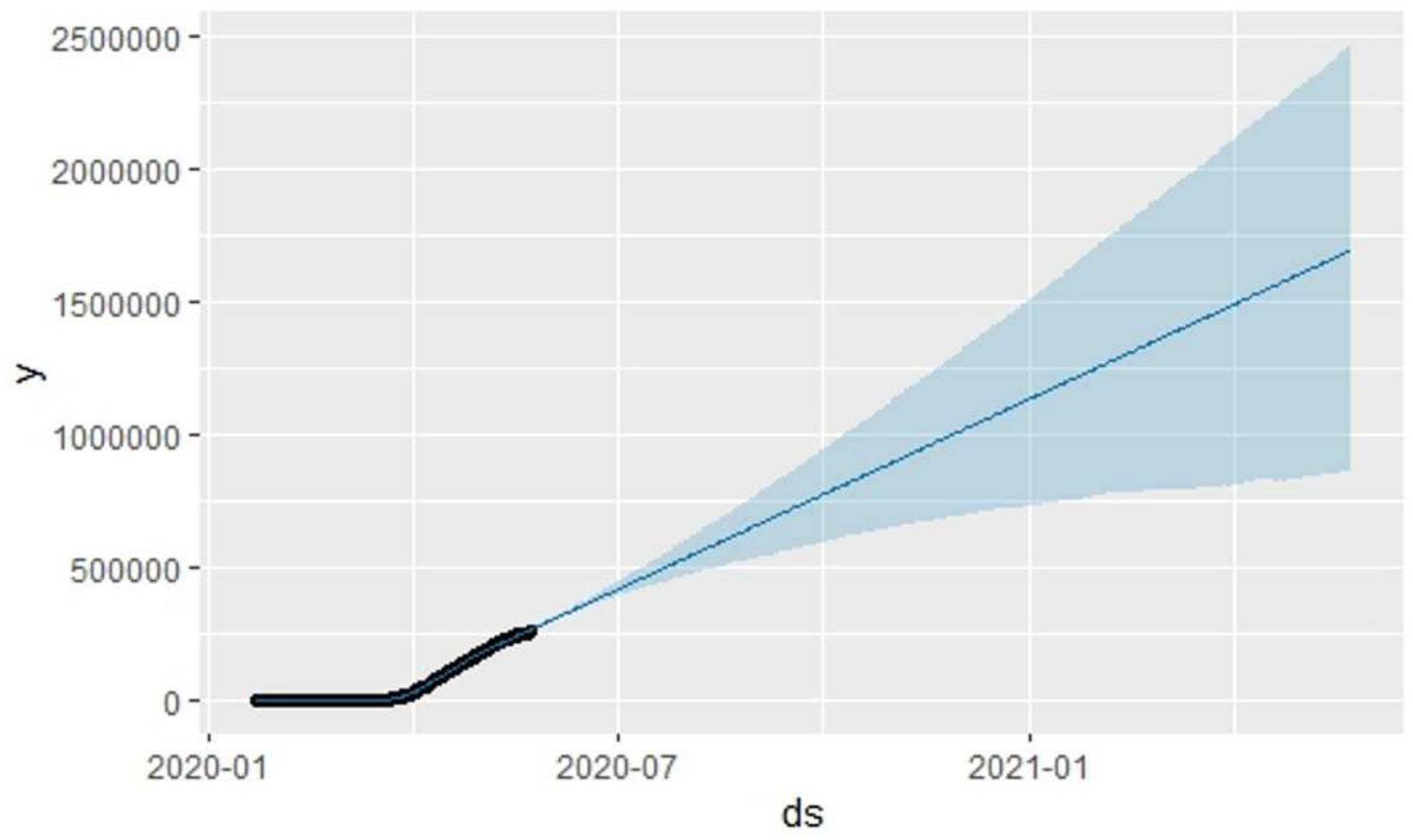

\section{Figure 22}

Forcasting by Prophet for the next year (Confirmed cases in United Kingdom)

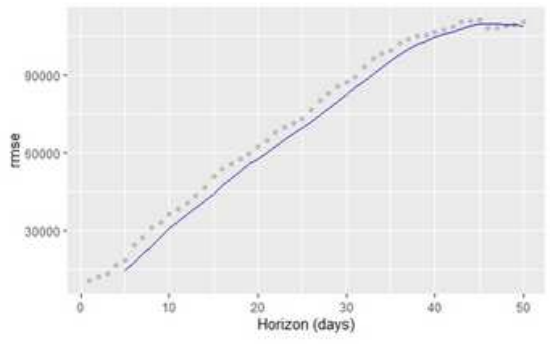

(a) United Kingdom

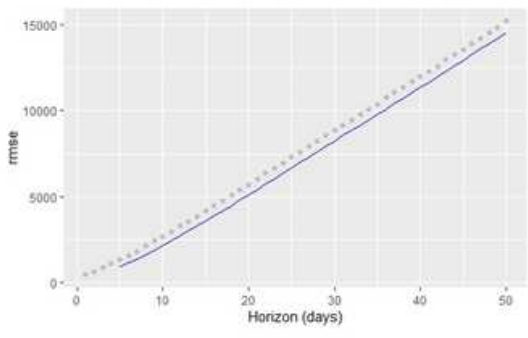

(b) Australia

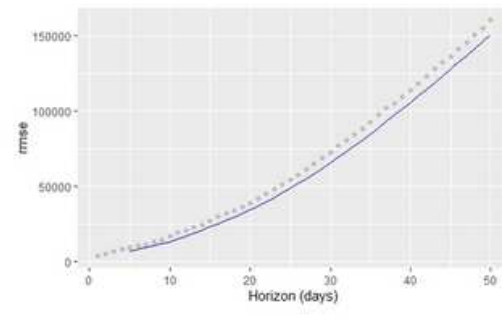

(C) Italy

\section{Figure 23}

Visualization of performance metric for Prophet for the countries (considering RMSE)

\section{Supplementary Files}

This is a list of supplementary files associated with this preprint. Click to download. 
- Supplementarymaterials.docx 